Homology, Homotopy and Applications, vol.18(1), 2016, pp.1-23

\title{
HOMOLOGY CYCLES IN MANIFOLDS WITH LOCALLY STANDARD TORUS ACTIONS
}

\author{
ANTON AYZENBERG
}

\author{
(communicated by Donald M. Davis)
}

\begin{abstract}
We describe the homology of a closed manifold $X$ with a locally standard action of a half-dimensional torus under the assumption that proper faces of its orbit space $Q$ are acyclic and the free part of action is trivial. There are three types of homology classes in $X$ : (1) classes of face submanifolds, (2) $k$-dimensional classes of $Q$ lifted to $X$ and swept by actions of subtori of dimensions $<k$, and (3) relative $k$-classes of $Q$ modulo $\partial Q$ lifted, in appropriate way, to $X$ and swept by actions of subtori of dimensions $\geqslant k$. The submodule spanned by face classes is an ideal in $H_{*}(X)$ with respect to the intersection product. As a ring it is isomorphic to $\left(\mathbb{Z}\left[S_{Q}\right] / \Theta\right) / W$, where $\mathbb{Z}\left[S_{Q}\right]$ is the face ring of the Buchsbaum simplicial poset dual to $Q, \Theta$ is an ideal generated by a linear system of parameters, and $W$ is a submodule lying in the socle of $\mathbb{Z}\left[S_{Q}\right] / \Theta$. The intersection product in homology is described in terms of the product in the face ring and intersection products on the orbit space and on the torus. Manifolds with torus actions provide a topological interpretation for the results of Novik and Swartz concerning socles of Buchsbaum face rings.
\end{abstract}

\section{Introduction}

An action of a compact torus $T^{n}$ on a smooth compact manifold $M$ of dimension $2 n$ is called locally standard if it is locally isomorphic to the standard action of $T^{n}$ on $\mathbb{C}^{n}$. The orbit space $Q=M / T^{n}$ is a manifold with corners whose open $k$ dimensional faces correspond to $k$-dimensional orbits of the action. If $Q$ is a simple polytope, the manifold $M$ is called quasitoric. The theory of such manifolds was created by Davis and Januszkiewicz [8], and further developed in many other works. Up to equivariant homeomorphism, a quasitoric manifold is determined by a socalled characteristic pair, which consists of a simple polytope (an orbit space) and

The author was supported by the JSPS postdoctoral fellowship for foreign researchers.

Received May 11, 2015, revised July 2, 2015, July 31, 2015; published on December 28, 2015.

2010 Mathematics Subject Classification: 57N65, 55N45, 55R91, 13F55, 13F50, 05E45, 06A07, 16W50, $13 \mathrm{H} 10$.

Key words and phrases: locally standard torus action, orbit type filtration, face submanifold, characteristic submanifold, intersection product, face ring, Buchsbaum simplicial poset, socle of a module. Article available at http://dx.doi.org/10.4310/HHA.2016.v18.n1.a1

Copyright (C) 2015, Anton Ayzenberg. Permission to copy for private use granted. 
a characteristic function (an information on stabilizers of the action). Yoshida [16] proved a natural extention of this classification: every manifold with locally standard torus action is equivariantly homeomorphic to the quotient model $X=Y / \sim$, where $Y$ is a principal $T^{n}$-bundle over $Q$ and $\sim$ is an equivalence relation determined by the characteristic function.

Quasitoric manifolds are topological counterparts of several familiar notions, such as complete smooth toric varieties or toric symplectic manifolds, and topology of such spaces is well studied. However, several constructions had appeared in differential geometry in the recent years, providing examples of manifolds with locally standard actions that are not quasitoric. These constructions include toric origami manifolds and toric log-symplectic manifolds. Thus there seems to be a need in the better understanding of topology in a larger classes of examples.

This paper is the third in a series of works where we study the topology of $X$ under the assumption that proper faces of the orbit space are acyclic and $Y$ is a trivial bundle - that is, $Y=Q \times T^{n}$. Previous papers $[\mathbf{1}, \mathbf{2}]$ were devoted to the homological spectral sequence associated with the filtration of orbit types on $X$. Here we discuss the geometrical structure of homology classes on $X$ and linear relations on them.

In the case when all faces of $Q$, including $Q$ itself, are acyclic (which is a slight generalization of the quasitoric case), the topology of the corresponding manifold $X$ is known [9]. In this case the equivariant cohomology $H_{T}^{*}(X ; \mathbb{Z})$ is isomorphic to the face ring $\mathbb{Z}\left[S_{Q}\right]$, where $S_{Q}$ is a simplicial poset dual to the orbit space. As a ring, equivariant cohomology is generated by equivariant classes, Poincare dual to face submanifolds of $X$, and these generators correspond to the standard generators of the face ring. The spectral sequence of the Borel fibration $E T^{n} \times_{T} X \rightarrow B T^{n}$ collapses at a second page, and the fiber inclusion $\iota: X \hookrightarrow E T^{n} \times_{T} X$ induces a surjective ring homomorphism $\iota^{*}: H_{T}^{*}(X ; \mathbb{Z}) \rightarrow H^{*}(X ; \mathbb{Z})$, whose kernel is the image of $H^{+}\left(B T^{n} ; \mathbb{Z}\right)$ under $\pi^{*}$. Thus $H^{*}(X ; \mathbb{Z}) \cong \mathbb{Z}\left[S_{Q}\right] /\left(\theta_{1}, \ldots, \theta_{n}\right)$, where $\theta_{i}$ are the images of the generators $v_{i}$ of the ring $H^{*}\left(B T^{n} ; \mathbb{Z}\right) \cong \mathbb{Z}\left[v_{1}, \ldots, v_{n}\right]$. The sequence $\left(\theta_{1}, \ldots, \theta_{n}\right)$ is a linear system of parameters in $\mathbb{Z}\left[S_{Q}\right]$. The poset $S_{Q}$ is Cohen-Macaulay, and thus $\left(\theta_{1}, \ldots, \theta_{n}\right)$ is a regular sequence, and we have $\operatorname{dim} H^{2 k}(X)=h_{k}\left(S_{Q}\right)$, while the cohomology in odd degrees vanishes.

In the case when only proper faces of $Q$ are acyclic, this approach does not work. The spectral sequence of the Borel fibration does not collapse at a second page. There still exists a ring homomorphism $H_{T}^{*}(X ; \mathbb{Z}) /\left(\pi^{*} H^{+}\left(B T^{n} ; \mathbb{Z}\right)\right) \rightarrow H^{*}(X ; \mathbb{Z})$, but it is neither injective nor surjective.

However, there is an apparent connection between topology of spaces with torus actions and the theory of face rings even in more general situations. In [3] we proved the ring isomorphism

$$
H_{T}^{*}(X ; \mathbb{Z}) \cong \mathbb{Z}\left[S_{Q}\right] \oplus H^{*}(Q ; \mathbb{Z}),
$$

where the degree 0 components of summands are identified.

When all proper faces of $Q$ are acyclic, the dual simplicial poset $S_{Q}$ is Buchsbaum. A standard tool in combinatorics and commutative algebra devised to study Buchsbaum simplicial posets is the $h^{\prime}$-vector. By definition, $h^{\prime}$-numbers of a Buchsbaum simplicial poset $S$ are the dimensions of homogeneous components of the quotient algebra $\mathbb{k}[S] /\left(\theta_{1}, \ldots, \theta_{n}\right)$, where $\theta_{1}, \ldots, \theta_{n}$ is a linear system of parameters. These 
numbers do not depend on the system of parameters, and can be expressed in terms of the ordinary $h$-numbers and Betti numbers of $S$ according to Schenzel [14] (for simplicial complexes), and Novik-Swartz [11] (for simplicial posets); see formula (3), below.

Previously we proved that $\operatorname{dim}\left(E_{X}\right)_{q, q}^{2}=h_{n-q}^{\prime}\left(S_{Q}\right)$, where $\left(E_{X}\right)_{*, *}^{*}$ is the spectral sequence in homology associated with the orbit type filtration on $X$. In this paper we describe the geometrical structure of $H_{*}(X)$.

Theorem 1.1. Homology classes of $X$ are of three different types:

1. The classes of face submanifolds. We call them face classes.

2. The classes, represented by $k$-cycles of $Q$, lifted to $X$ and swept by actions of $l$-dimensional subtori with $l<k$. These classes will be called spine classes.

3. The classes, represented by relative $k$-cycles of $Q$ modulo $\partial Q$ with $k<n$, lifted in an appropriate way to $X$ and swept by actions of l-dimensional subtori with $l \geqslant k$. These classes will be called diaphragm classes.

Linear relations on face classes are of two types: the relations appearing in the ring $\mathbb{Z}\left[S_{Q}\right] /\left(\theta_{1}, \ldots, \theta_{n}\right)$, and additional relations lying in a socle of $\mathbb{Z}\left[S_{Q}\right] /\left(\theta_{1}, \ldots, \theta_{n}\right)$.

Intersections of face classes are encoded by the product in the face ring of $S_{Q}$. Proper face classes span the ideal of $H_{*}(X ; \mathbb{Z})$ with respect to the intersection product. Intersections of other classes are described via the intersection products on $Q$ and $T^{n}$.

More precise statements are given by Propositions 2.9, 4.1, 5.6, 6.2, 6.3, and 6.4. Face classes and the elements of $H_{k}(Q, \partial Q)$ swept by the action of the whole group $T^{n}$ are equivariant. This gives an independent geometrical evidence for the formula (1).

Additional relations on face classes are represented by certain elements of the group $\mathbb{k}\left[S_{Q}\right] /\left(\theta_{1}, \ldots, \theta_{n}\right)$. These elements have a specific form, and in Theorem 4.6 we prove that all elements of such form lie in the socle of $\mathbb{k}\left[S_{Q}\right] /\left(\theta_{1}, \ldots, \theta_{n}\right)$. This gives a geometrical intuition behind the result of Novik and Swartz [11].

The paper may be briefly outlined as follows. In Section 2 we review the basic definitions and our previous results. Section 3 contains technical preparations for the subsequent sections. In particular, we discuss orientation issues and prove Lemma 3.3, which is used in many places throughout the paper. Section 4 is devoted to linear relations on face classes in $H_{*}(X)$. We give precise formulas for these relations, and in particular describe the kernel of the composite map

$$
\mathbb{Z}\left[S_{Q}\right] /\left(\theta_{1}, \ldots, \theta_{n}\right) \hookrightarrow H_{T}^{*}(X ; \mathbb{Z}) /\left(\pi^{*} H^{+}\left(B T^{n} ; \mathbb{Z}\right)\right) \rightarrow H^{*}(X ; \mathbb{Z}) .
$$

In Section 5 we realize non-face classes of $X$ as embedded pseudomanifolds. These geometrical constructions imply a partial description of intersection theory on $X$, which is given in Section 6. Two examples are discussed in Section 7. A very particular 4-dimensional example is worked out, and the reader is encouraged to refer to this example while reading the other parts of the paper. The second example is more general: the technique is applied to the class of orientable toric origami manifolds with acyclic proper faces of the orbit space, and we rediscover some results of [3] by a new method. In the last section we introduce a supplementary space $\widehat{X}$ that can be considered as a $T^{n}$-invariant tubular neighborhood of the union of characteristic submanifolds in $X$. By using the intersection theory on $\widehat{X}$, we prove that certain elements of $\mathbb{k}\left[S_{Q}\right] /\left(\theta_{1}, \ldots, \theta_{n}\right)$ lie in the socle. 


\section{Acknowledgments}

The author thanks Ryota Okazaki for a nice introduction to the theory of Buchsbaum complexes, and Shintaro Kuroki, who suggested the existence of possible connections between $h^{\prime \prime}$-numbers and toric spaces.

\section{Definitions and previous results}

\subsection{Manifolds with locally standard actions}

An action of $T^{n}$ on a (compact connected smooth) manifold $M^{2 n}$ is called locally standard if $M$ has an atlas of $T^{n}$-invariant charts, each equivalent to an open $T^{n}$ invariant subset of the standard action of $T^{n}$ on $\mathbb{C}^{n} \cong \mathbb{R}^{2 n}$, up to some automorphism of torus. We refer to the monograph of Buchstaber and Panov [6] or to the work of Yoshida [16] for details relevant to our study. The orbit space of a locally standard action is a compact connected $n$-dimensional manifold with corners with the property that every codimension $k$ face of $Q$ belongs to exactly $k$ facets (such manifolds with corners were called nice by Masuda and Panov [9], or manifolds with faces elsewhere).

Definition 2.1. A finite partially ordered set (poset) $S$ is called simplicial if (1) there exists a unique minimal element $\hat{0} \in S$, and (2) for each element $J \in S$ the interval $\{I \in S \mid I \leqslant J\}$ is isomorphic to the poset of faces of a $k$-simplex for some number $k$, called the dimension of $J$.

The elements of $S$ are called simplices. Simplices of dimension 0 are called vertices. The number $|I|=\operatorname{dim} I+1$ is equal to the number of vertices of $I$ and is called the rank of $I$. The set of vertices of a simplicial poset $*$ or a simplex $*$ will be denoted by $\operatorname{Vert}(*)$.

Every manifold $Q$ with corners determines a poset $S_{Q}$ whose elements are the faces of $Q$ ordered by reversed inclusion. When $Q$ is a nice connected manifold with corners, $S_{Q}$ is a simplicial poset. For convenience we denote abstract elements of $S_{Q}$ by $I, J$, etc., and the corresponding faces of $Q$ by $F_{I}, F_{J}$, etc. There holds $\operatorname{dim} F_{I}=n-|I|$, where $n$ is the dimension of $Q$. The minimal element of $S_{Q}$ corresponds to the maximal face of $Q$ - that is, $Q$ itself - and vertices of $S_{Q}$ correspond to facets of $Q$. The set of facets of $Q$ will be denoted by $\operatorname{Fac}(Q)$.

Let $Q$ be the orbit space of locally standard action, and let $x$ be a point in the interior of a facet $F \in \operatorname{Fac}(Q)$. Then the stabilizer of $x$, denoted by $\lambda(F)$, is a 1 dimensional toric subgroup in $T^{n}$. If $F_{I}$ is a codimension $k$ face of $Q$ lying in the facets $F_{1}, \ldots, F_{k} \in \operatorname{Fac}(Q)$, then the stabilizer of $x \in F_{I}^{\circ}$ is the $k$-dimensional torus $T_{I}=$ $\lambda\left(F_{1}\right) \times \cdots \times \lambda\left(F_{k}\right) \subset T^{n}$, where the product is free inside $T^{n}$. This puts a specific restriction on subgroups $\lambda(F)$. In general, a map

$$
\lambda: \operatorname{Fac}(Q) \rightarrow\left\{1 \text {-dimensional toric subgroups of } T^{n}\right\}
$$

is called a characteristic function if, whenever facets $F_{1}, \ldots, F_{k}$ have a non-empty intersection, the map

$$
\lambda\left(F_{1}\right) \times \cdots \times \lambda\left(F_{k}\right) \rightarrow T^{n},
$$

induced by inclusions $\lambda\left(F_{i}\right) \hookrightarrow T^{n}$, is injective and splits. This condition is called (*)-condition (the terminology goes back to Davis and Januszkiewicz [8]). Let $i \in$ 
$\operatorname{Vert}\left(S_{Q}\right)$ be the vertex of $S_{Q}$, and $T_{i}=\lambda\left(F_{i}\right)$ be the value of characteristic function. Let $\omega_{i} \in H_{1}\left(T^{n} ; \mathbb{k}\right) \cong \mathbb{k}^{n}$ denote the fundamental class of 1-dimensional subtorus $T_{i}$. This class is defined uniquely up to sign. Here $\mathbb{k}$ is the ground ring, and is either $\mathbb{Z}$ or a field.

Let $\mu: M \rightarrow Q$ be the projection to the orbit space. The free part of the action has the form $\left.\mu\right|_{Q^{\circ}}: \mu^{-1}\left(Q^{\circ}\right) \rightarrow Q^{\circ}$, where $Q^{\circ}=Q \backslash \partial Q$ is the interior of the manifold with corners. The free part is a principal torus bundle over $Q^{\circ}$. It can be uniquely extended over $Q$ and determines a principal $T^{n}$-bundle $\rho: Y \rightarrow Q$. Therefore, any manifold with locally standard action defines three objects: the nice manifold with corners $Q$, the principal torus bundle $\rho: Y \rightarrow Q$, and the characteristic function $\lambda$. One can recover $M$, up to equivariant homeomorphism, from these data by the following standard construction.

Construction 2.2 (Quotient construction). Let $\rho: Y \rightarrow Q$ be a principal $T^{n}$-bundle over a nice manifold with corners, and let $\lambda$ be a characteristic function on $\operatorname{Fac}(Q)$. Consider the space $X \stackrel{\text { def }}{=} Y / \sim$, where $y_{1} \sim y_{2}$ if and only if $\rho\left(y_{1}\right)=\rho\left(y_{2}\right) \in F_{I}^{\circ}$ for some face $F_{I}$ of $Q$, and $y_{1}, y_{2}$ lie in the same $T_{I}$-orbit of the $T^{n}$-action on $Y$. Let $f: Y \rightarrow X$ denote the quotient map.

Every manifold $M$ with locally standard torus action is equivariantly homeomorphic to its model $X$ [16, Cor. 2]. In the rest of the paper we use the model $X$ instead of $M$.

Remark 2.3. We work with a smooth manifold with corners $Q$ and smooth manifolds $X \cong M$, but this is done basically to simplify the exposition. The quotient model $X=\left(Q \times T^{n}\right) / \sim$ can obviously be defined for a larger class of spaces. If $Q$ is a homology manifold with a simple stratification of the boundary, and the faces are homology manifolds with boundaries as well, then $X$ is a closed homology manifold. The results of the paper are valid in this setting.

\subsection{Filtrations}

There are natural topological filtrations on $Q, Y$, and $X$. Namely, $Q_{k} \subseteq Q$ is the union of $k$-dimensional faces of $Q, Y_{k}=\rho^{-1}\left(Q_{k}\right) \subseteq Y$, and $X_{k}=f\left(Y_{k}\right) \subset X$ is the union of toric orbits of dimension at most $k$. The maps $\mu: X \rightarrow Q, \rho: Y \rightarrow Q$, and $f: Y \rightarrow X$ respect these filtrations. The homological spectral sequences produced by these filtrations are denoted by $\left(E_{Q}\right)_{*, *}^{*},\left(E_{Y}\right)_{*, *}^{*}$, and $\left(E_{X}\right)_{*, *}^{*}$. The map $f$ induces the morphism of spectral sequences $f_{*}^{r}:\left(E_{Y}\right)^{r} \rightarrow\left(E_{X}\right)^{r}$.

The subsets $\rho^{-1}\left(F_{I}\right) \subset Y$ and $\mu^{-1}\left(F_{I}\right) \subset X$, which cover the face $F_{I} \subset Q$, are denoted by $Y_{I}$ and $X_{I}$, respectively. Note that the subset $X_{I}$ is a closed submani-

fold of $X$ of codimension $2|I|$. It is called a face submanifold. Face submanifolds of codimension 2 are called characteristic submanifolds. They correspond to facets of $Q$.

The first page of $\left(E_{Q}\right)_{*, *}^{*}$ has the form

$$
\left(E_{Q}\right)_{p, q}^{1}=H_{p+q}\left(Q_{p}, Q_{p-1}\right) \cong \bigoplus_{I \in S_{Q}, \operatorname{dim} F_{I}=p} H_{p+q}\left(F_{I}, \partial F_{I}\right)
$$


and the first differential $\left(d_{Q}\right)^{1}$ is the sum of the maps

$$
\begin{aligned}
m_{I, J}^{q}: H_{q+\operatorname{dim} F_{I}}\left(F_{I}, \partial F_{I}\right) & \rightarrow H_{q+\operatorname{dim} F_{I}-1}\left(\partial F_{I}\right) \rightarrow \\
& \rightarrow H_{q+\operatorname{dim} F_{I}-1}\left(\partial F_{I}, \partial F_{I} \backslash F_{J}^{\circ}\right) \cong H_{q+\operatorname{dim} F_{J}}\left(F_{J}, \partial F_{J}\right),
\end{aligned}
$$

defined for every face $F_{I}$ and $F_{J} \in \operatorname{Fac}\left(F_{I}\right)$. Here the first map is the connecting homomorphism in the homology exact sequence of $\left(F_{I}, \partial F_{I}\right)$, and the last isomorphism is due to excision. If the coefficient ring in the notation of (co)homology is omitted, it is supposed to be the ground ring $\mathbb{k}$.

\subsection{The case of acyclic proper faces}

Further on we impose two restrictions on $X$ mentioned in the introduction. First, we assume that $Q$ is an orientable manifold and all its proper faces are acyclic (over $\mathbb{k}$ ). Second, the principal torus bundle $Y \rightarrow Q$ is assumed to be trivial. Thus $X=$ $\left(Q \times T^{n}\right) / \sim$. The following propositions were proved in $[\mathbf{1}, \mathbf{2}]$.

Proposition 2.4. The poset $S_{Q}$ is a Buchsbaum simplicial poset (over $\mathbb{k}$ ). Moreover, the geometrical realization $\left|S_{Q}\right|$ is an orientable homology manifold.

Proposition 2.5. There exists a homological spectral sequence $\left(\dot{E}_{Q}\right)_{p, q}^{r} \Rightarrow H_{p+q}(Q)$, $\left(\dot{d}_{Q}\right)^{r}:\left(\dot{E}_{Q}\right)_{p, q}^{r} \rightarrow\left(\dot{E}_{Q}\right)_{p-r, q+r-1}^{r}$ with the following properties:

1. $\left(\dot{E}_{Q}\right)^{1}=H\left(\left(E_{Q}\right)^{1}, d_{Q}^{-}\right)$, where the differential $d_{Q}^{-}:\left(E_{Q}\right)_{p, q}^{1} \rightarrow\left(E_{Q}\right)_{p-1, q}^{1}$ coincides with $\left(d_{Q}\right)^{1}$ for $p<n$, and vanishes otherwise.

2. The module $\left(\dot{E}_{Q}\right)_{*, *}^{r}$ coincides with $\left(E_{Q}\right)_{*, *}^{r}$ for $r \geqslant 2$.

3. $\left(\dot{E}_{Q}\right)_{p, q}^{1}=\left\{\begin{array}{l}H_{p}(\partial Q), \text { if } q=0, p<n ; \\ H_{q+n}(Q, \partial Q), \text { if } p=n, q \leqslant 0 ; \\ 0, \text { otherwise. }\end{array}\right.$

4. Nontrivial differentials for $r \geqslant 1$ have the form $\left(\dot{d}_{Q}\right)^{r}:\left(\dot{E}_{Q}\right)_{n, 1-r}^{r} \rightarrow\left(\dot{E}_{Q}\right)_{n-r, 0}^{r}$ and coincide with the connecting homomorphisms $\delta_{n+1-r}: H_{n+1-r}(Q, \partial Q) \rightarrow$ $H_{n-r}(\partial Q)$ in the long exact sequence of the pair $(Q, \partial Q)$.

Let $\Lambda_{*}$ denote the homology module of a torus: $\Lambda_{*}=\bigoplus_{s} \Lambda_{s}, \Lambda_{s}=H_{s}\left(T^{n}\right)$.

Proposition 2.6. There exists a homological spectral sequence $\left(\dot{E}_{Y}\right)_{p, q}^{r} \Rightarrow H_{p+q}(Y)$ such that:

1. $\left(\dot{E}_{Y}\right)^{1}=H\left(\left(E_{Y}\right)^{1}, d_{Y}^{-}\right)$, where the differential $d_{Y}^{-}:\left(E_{Y}\right)_{p, q}^{1} \rightarrow\left(E_{Y}\right)_{p-1, q}^{1}$ coincides with $\left(d_{Y}\right)^{1}$ for $p<n$, and vanishes otherwise.

2. $\left(\dot{E}_{Y}\right)^{r}=\left(E_{Y}\right)^{r}$ for $r \geqslant 2$.

3. $\left(\dot{E}_{Y}\right)_{p, q}^{r}=\bigoplus_{q_{1}+q_{2}=q}\left(\dot{E}_{Q}\right)_{p, q_{1}}^{r} \otimes \Lambda_{q_{2}}$ and $\left(\dot{d}_{Y}\right)^{r}=\left(\dot{d}_{Q}\right)^{r} \otimes \operatorname{id}_{\Lambda}$ for $r \geqslant 1$.

Proposition 2.7. There exists a homological spectral sequence $\left(\dot{E}_{X}\right)_{p, q}^{r} \Rightarrow H_{p+q}(X)$ and the morphism of spectral sequences $\dot{f}_{*}^{r}:\left(\dot{E}_{Y}\right)_{*, *}^{r} \rightarrow\left(\dot{E}_{X}\right)_{*, *}^{r}$ such that:

1. $\left(\dot{E}_{X}\right)^{1}=H\left(\left(E_{X}\right)^{1}, d_{X}^{-}\right)$where the differential $d_{X}^{-}:\left(E_{X}\right)_{p, q}^{1} \rightarrow\left(E_{X}\right)_{p-1, q}^{1}$ coincides with $\left(d_{X}\right)^{1}$ for $p<n$, and vanishes otherwise. The map $\dot{f}_{*}^{1}$ is induced by $f_{*}^{1}:\left(E_{Y}\right)^{1} \rightarrow\left(E_{X}\right)^{1}$. 
2. $\left(\dot{E}_{X}\right)^{r}=\left(E_{X}\right)^{r}$ and $\dot{f}_{*}^{r}=f_{*}^{r}$ for $r \geqslant 2$.

3. $\left(E_{X}\right)_{p, q}^{1}=\left(\dot{E}_{X}\right)_{p, q}^{1}=0$ for $p<q$.

4. $\dot{f}_{*}^{1}:\left(\dot{E}_{Y}\right)_{p, q}^{1} \rightarrow\left(\dot{E}_{X}\right)_{p, q}^{1}$ is an isomorphism for $p>q$ and injective for $p=q$.

5. As a consequence of previous items, for $r \geqslant 1$, the differentials $\left(\dot{d}_{X}\right)^{r}$ are either isomorphic to $\left(\dot{d}_{Y}\right)^{r}$ (when they hit the cells with $p>q$ ), or isomorphic to the composition of $\left(\dot{d}_{Y}\right)^{r}$ with $\dot{f}_{*}^{r}$ (when they hit the cells with $p=q$ ), or zero (otherwise).

6. The ranks of diagonal terms at a second page are the $h^{\prime}$-numbers of the poset $S_{Q}$ dual to the orbit space: $\operatorname{dim}\left(\dot{E}_{X}\right)_{q, q}^{2}=\operatorname{dim}\left(E_{X}\right)_{q, q}^{2}=h_{n-q}^{\prime}\left(S_{Q}\right)$.

Recall the combinatorial definition of $h^{\prime}$-numbers.

Definition 2.8. Let $S$ be a pure simplicial poset, $\operatorname{dim} S=n-1$. Let $f_{k}$ be the the number of $k$-dimensional simplices in $S$. An array $\left(f_{-1}=1, f_{0}, \ldots, f_{n-1}\right)$ is called the $f$-vector of $S$. $h$-numbers are defined by the formula

$$
h_{0} s^{n}+h_{1} s^{n-1}+\cdots+h_{n}=f_{-1}(s-1)^{n}+f_{0}(s-1)^{n-1}+\cdots+f_{n-1} .
$$

Let $\widetilde{\beta}_{k}(S)=\operatorname{dim} \widetilde{H}_{k}(S) \cdot h^{\prime}$-numbers are defined by

$$
h_{k}^{\prime}=h_{k}+\left(\begin{array}{l}
n \\
k
\end{array}\right)\left(\sum_{j=1}^{k-1}(-1)^{k-j-1} \widetilde{\beta}_{j-1}(S)\right) \text { for } 0 \leqslant k \leqslant n .
$$

Propositions 2.5-2.7 yield the description of $H_{*}(X)$. Let $H_{k, l}(Y)$ denote the $\mathbb{k}$ module $H_{k}(Q) \otimes \Lambda_{l}$. By Künneth's formula, $H_{j}(Y) \cong \bigoplus_{k+l=j} H_{k, l}(Y)$.

Proposition 2.9. Over a field, there exists a decomposition $H_{j}(X) \cong \bigoplus_{k+l=j} H_{k, l}(X)$ and $\mathbb{k}$-module homomorphisms $f_{*}: H_{k, l}(Y) \rightarrow H_{k, l}(X)$ with the following properties:

1. If $k>l$, then $f_{*}: H_{k, l}(Y) \rightarrow H_{k, l}(X)$ is an isomorphism. In particular, $H_{k, l}(X) \cong H_{k}(Q) \otimes \Lambda_{l}$.

2. If $k<l$, there exists an isomorphism $H_{k, l}(X) \cong H_{k}(Q, \partial Q) \otimes \Lambda_{l}$.

3. If $k<n$, the module $H_{k, k}(X)$ fits in the short exact sequence

$$
0 \rightarrow\left(\dot{E}_{X}\right)_{k, k}^{\infty} \rightarrow H_{k, k}(X) \rightarrow H_{k}(Q, \partial Q) \otimes \Lambda_{k} \rightarrow 0 .
$$

4. $H_{n, n}(X) \cong \mathbb{k}$.

5. Bigraded Poincare duality holds: $H_{k, l}(X) \cong H_{n-k, n-l}(X)$.

Later, in Section 5 , we will show that this proposition remains valid over $\mathbb{Z}$.

\section{Preliminary computations}

\subsection{Orientations}

We use the notation $I \stackrel{k}{<} J$ whenever simplices $I, J \in S$ satisfy $I<J$ and $|J|-$ $|I|=k$. For each pair $I \stackrel{2}{<} J$, there are exactly two simplices $J^{\prime} \neq J^{\prime \prime}$ in between:

$I \stackrel{1}{<} J^{\prime}, J^{\prime \prime} \stackrel{1}{<} J$. For every simplicial poset, there exists a "sign convention" that means 
that an incidence number $[J: I]= \pm 1$ is associated with any pair $I \stackrel{1}{<} J \in S$, and the relation $\left[J: J^{\prime}\right] \cdot\left[J^{\prime}: I\right]+\left[J: J^{\prime \prime}\right] \cdot\left[J^{\prime \prime}: I\right]=0$ holds for any $I \stackrel{2}{<} J$.

The choice of a sign convention is equivalent to the choice of orientations of all nonempty simplices. By the orientation of a simplex in an abstract simplicial poset we mean the rule that tells whether a given total ordering of the vertices of $I$ is positive or negative, so that even permutations of an ordering preserve the sign and odd permutations change it. If $I \stackrel{1}{<} J$, then there is exactly one vertex $i$ of $J$ that is not in $I$. Given the orientations of simplices $I$ and $J$, and given some positive ordering $i_{1}<\cdots<i_{s}$ of the vertices of $I$, we set $[J: I]$ to be +1 if $i<i_{1}<\cdots<i_{s}$ is a positive ordering on $\operatorname{Vert}(J)$, and -1 otherwise. The construction works in the opposite direction in an obvious way: incidence signs determine orientations of all simplices by induction.

Fix arbitrary orientations of the orbit space $Q$ and the torus $T^{n}$. Together they determine an orientation of $Y=Q \times T^{n}$ and $X=Y / \sim$. Also choose an omniorientation, which means the orientations of all characteristic submanifolds. A choice of an omniorientation determines the characteristic values $\omega_{i} \in H_{1}\left(T^{n} ; \mathbb{Z}\right)$ without ambiguity of sign. To perform explicit calculations with the spectral sequences $\left(\dot{E}_{X}\right)^{*}$ and $\left(\dot{E}_{Y}\right)^{*}$ we also need to orient all faces of $Q$.

Construction 3.1. An orientation of a simplex $I \in S_{Q}$ determines an orientation of a face $F_{I} \subset Q$ by the following convention. Let $i_{1}, \ldots, i_{n-q}$ be the vertices of $I$, listed in a positive order. The face $F_{I}$ lies in the intersection of facets $F_{i_{1}}, \ldots, F_{i_{n-q}}$. The normal bundles $\nu_{i}$ to facets $F_{i}$ have natural orientations, in which inward normal directions are positive. Orient $F_{I}$ in such way that $T_{x} F_{I} \oplus \nu_{i_{1}} \oplus \cdots \oplus \nu_{i_{n-q}} \cong T_{x} Q$ is positive with respect to the orientation of $Q$. Thus there are distinguished elements $\left[F_{I}\right] \in H_{\operatorname{dim} F_{I}}\left(F_{I}, \partial F_{I}\right)$. It can be seen that the homomorphism

$$
m_{I, J}^{0}: H_{\operatorname{dim} F_{I}}\left(F_{I}, \partial F_{I}\right) \rightarrow H_{\operatorname{dim} F_{J}}\left(F_{J}, \partial F_{J}\right)
$$

$(\operatorname{see}(2)) \operatorname{maps}\left[F_{I}\right]$ to $[J: I] \cdot\left[F_{J}\right]$.

A choice of omniorientation and orientations of simplices determines an orientation of each orbit $T^{n} / T_{I}$ as follows.

Construction 3.2. Let $i_{1}, \ldots, i_{n-q}$ be the vertices of $I$, listed in a positive order. The module $H_{1}\left(T^{n} / T_{I}\right)$ is naturally identified with $\Lambda_{1} / L_{I}$, where $L_{I}$ is a submodule of $\Lambda_{1}=H_{1}\left(T^{n}\right)$ generated by $\omega_{i_{1}}, \ldots, \omega_{i_{n-q}}$. We say that the basis $\left[\gamma_{1}\right], \ldots,\left[\gamma_{q}\right] \in$ $H_{1}\left(T^{n} / T_{I}\right),\left[\gamma_{l}\right]=\gamma_{l}+L_{I}$ determines the positive orientation of $H_{1}\left(T^{n} / T_{I}\right)$ if the basis $\left(\omega_{i_{1}}, \ldots, \omega_{i_{n-q}}, \gamma_{1}, \ldots, \gamma_{q}\right)$ determines the positive orientation of $\Lambda_{1}$. This orientation of $T^{n} / T_{I}$ determines a distinguished fundamental class $\Omega_{I} \in H_{q}\left(T^{n} / T_{I}\right)$.

The omniorientation and the orientation of $S$ together determine the class of each face submanifold: $\left[X_{I}\right]=\left[F_{I}\right] \otimes \Omega_{I}$. Note that both orientations $\left[F_{I}\right]$ and $\left[\Omega_{I}\right]$ depend on the orientation of $I$ by construction. Thus $\left[X_{I}\right]$ does not actually depend on the sign convention on $S_{Q}$ and depends only on the omniorientation.

\subsection{Arithmetics of torus quotients}

Let us fix a coordinate representation of the torus $T^{n}=T^{(\{1\})} \times \cdots \times T^{(\{n\})}$, where each $T^{(\{j\})}$ is a 1-dimensional torus with a chosen orientation. For a subset $A=$ 
$\left\{j_{1}<\cdots<j_{q}\right\} \subseteq[n]$ we denote the coordinate subtorus $T^{\left(\left\{j_{1}\right\}\right)} \times \cdots \times T^{\left(\left\{j_{q}\right\}\right)} \subseteq T^{n}$ by $T^{(A)}$.

The coordinate splitting gives a positive basis $e_{1}, \ldots, e_{n}$ of the module $\Lambda_{1}=$ $H_{1}\left(T^{n}\right)$, where $e_{j}$ is the fundamental class of $T^{(\{j\})}$. For a vertex $i \in \operatorname{Vert}(S)$ let $\left(\lambda_{i, 1}, \ldots, \lambda_{i, n}\right)$ denote the coordinates of $\omega_{i}=\left[\lambda\left(F_{i}\right)\right] \in \Lambda_{1}$ in this basis.

Lemma 3.3. Let $I \in S_{Q} \backslash \hat{0}$ be a simplex with the vertices $\left\{i_{1}, \ldots, i_{n-q}\right\}$ listed in a positive order. Let $A=\left\{j_{1}<\cdots<j_{q}\right\} \subset[n]$ be a subset of indices, and let $e_{A}=e_{j_{1}} \wedge$ $\cdots \wedge e_{j_{q}} \in H_{q}\left(T^{n} ; \mathbb{Z}\right)$ be the fundamental class of the coordinate subtorus $T^{(A)} \subseteq T^{n}$. Consider the map $\varrho: T^{n} \rightarrow T^{n} / T_{I}$. Then $\varrho_{*}\left(e_{A}\right)=C_{I, A} \Omega_{I} \in H_{q}\left(T^{n} / T_{I} ; \mathbb{Z}\right)$ and the constant $C_{I, A}$ is equal to

$$
\operatorname{sgn}_{A} \operatorname{det}\left(\lambda_{i, j}\right)_{\substack{i \in\left\{i_{1}, \ldots, i_{n-q}\right\} \\ j \in[n] \backslash A}}
$$

where $\operatorname{sgn}_{A}= \pm 1$ depends only on $A \subset[n]$. When $q=0$, the constant $C_{I, A}$ equals \pm 1 depending on the positivity of the basis $\omega_{i_{1}}, \ldots, \omega_{i_{n}}$.

Proof. For $q=0$ the statement is obvious, so we assume $q \neq 0$. Choose arbitrary vectors $\gamma_{1}, \ldots, \gamma_{q}$ such that $\left(b_{l}\right)=\left(\omega_{i_{1}}, \ldots, \omega_{i_{n-q}}, \gamma_{1}, \ldots, \gamma_{q}\right)$ is a positive basis of the lattice $H_{1}\left(T^{n}, \mathbb{Z}\right)$. Thus $b_{l}=\mathbf{U} e_{l}$, where $\mathbf{U}$ is the matrix of the form

$$
\mathbf{U}=\left(\begin{array}{cccccc}
\lambda_{i_{1}, 1} & \cdots & \lambda_{i_{n-q}, 1} & * & \cdots & * \\
\lambda_{i_{1}, 2} & \cdots & \lambda_{i_{n-q}, 2} & * & \cdots & * \\
\vdots & \ddots & \vdots & \vdots & \ddots & \vdots \\
\lambda_{i_{1}, n} & \cdots & \lambda_{i_{n-q}, n} & * & \cdots & *
\end{array}\right)
$$

We have $\operatorname{det} \mathbf{U}=1$ since both bases are positive. Consider the inverse matrix $\mathbf{V}=$ $\mathbf{U}^{-1}$. We have

$$
e_{A}=e_{j_{1}} \wedge \cdots \wedge e_{j_{q}}=\sum_{M=\left\{\alpha_{1}<\ldots<\alpha_{q}\right\} \subset[n]} \operatorname{det}\left(\mathbf{V}_{j, \alpha}\right)_{j \in A} b_{\alpha \in M} b_{\alpha_{1}} \wedge \ldots \wedge b_{\alpha_{q}} .
$$

After taking the quotient $\Lambda /\left\langle\omega_{i_{1}}, \ldots, \omega_{i_{n-q}}\right\rangle$, all summands with $M \neq\{n-q+1, \ldots$ $\ldots, n\}$ vanish. When $M=\{n-q+1, \ldots, n\}$, the element $b_{n-q+1} \wedge \cdots \wedge b_{n}=\gamma_{1} \wedge$ $\cdots \wedge \gamma_{q}$ maps to $\Omega_{I}$. Thus

$$
C_{I, A}=\operatorname{det}\left(\mathbf{V}_{j, \alpha}\right)_{\substack{j \in A \\ \alpha \in\{n-q+1, \ldots, n\}}} .
$$

Now apply Jacobi's identity (see, e.g., [4]):

$$
\operatorname{det}\left(\mathbf{V}_{j, \alpha}\right)_{\substack{j \in A \\ \alpha \in\{n-q+1, \ldots, n\}}}=\frac{(-1)^{\mathrm{sgn}}}{\operatorname{det} \mathbf{U}} \operatorname{det}\left(\mathbf{U}_{r, s}\right)_{\substack{r \in\{1, \ldots, n-q\} \\ s \in[n] \backslash A}},
$$

where sgn $=\sum_{r=1}^{n-q} r+\sum_{s \in[n] \backslash A} s$. Since the first $n-q$ columns of $\mathbf{U}$ are exactly the vectors $\lambda_{i, j}$, this observation completes the proof.

\subsection{Face ring and a linear system of parameters}

Recall the definition of a face ring (for details refer to [15] or [5]). Let $I_{1} \vee I_{2}$ denote the set of least upper bounds of simplices $I_{1}, I_{2} \in S$, and let $I_{1} \cap I_{2} \in S$ denote the intersection of simplices (it is well defined and unique when $I_{1} \vee I_{2} \neq \varnothing$ ). 
Definition 3.4. The face ring $\mathbb{k}[S]$ is the quotient of the polynomial ring $\mathbb{k}\left[\left\{v_{I}\right\}\right]$, generated by variables $\left\{v_{I} \mid I \in S\right\}$, $\operatorname{deg} v_{I}=2|I|$, by the relations

$$
v_{I_{1}} \cdot v_{I_{2}}=v_{I_{1} \cap I_{2}} \cdot \sum_{J \in I_{1} \vee I_{2}} v_{J}, \quad v_{\varnothing}=1 .
$$

The sum over an empty set is assumed to be 0 .

Let $[m]=\{1, \ldots, m\}$ be the set of vertices of $S$, and let $\mathbb{k}[m]=\mathbb{k}\left[v_{1}, \ldots, v_{m}\right]$ be the graded ring of polynomials with $\operatorname{deg} v_{i}=2$. The ring homomorphism $\mathbb{k}[m] \rightarrow \mathbb{k}[S]$ sending $v_{i}$ to $v_{i}$ determines the structure of $\mathbb{k}[m]$-module on $\mathbb{k}[S]$.

Every characteristic function on $Q$ determines the set of linear forms $\left\{\theta_{1}, \ldots, \theta_{n}\right\} \subset$ $\mathbb{k}\left[S_{Q}\right]$, where $\theta_{j}=\sum_{i \in \operatorname{Vert}\left(S_{Q}\right)} \lambda_{i, j} v_{i}$. If $J \in S$ is a maximal simplex, i.e., $|J|=n$, then

$$
\text { the matrix }\left(\lambda_{i, j}\right)_{\substack{i \leqslant j \\ j \in[n]}} \text { is invertible over } \mathbb{k}
$$

by the $(*)$-condition. This condition is equivalent to the statement that the sequence $\left\{\theta_{1}, \ldots, \theta_{n}\right\}$ is a linear system of parameters in $\mathbb{k}[S][\mathbf{6}, \mathrm{Lm} .3 .5 .8]$. This sequence generates an ideal $\left(\theta_{1}, \ldots, \theta_{n}\right) \subset \mathbb{k}[S]$, which will be denoted by $\Theta$.

A face ring $\mathbb{k}[S]$ is an algebra with straightening law [15]. As a $\mathbb{k}$-module, it has an additive basis

$$
\left\{P_{\sigma}=v_{I_{1}} \cdot v_{I_{2}} \cdot \ldots \cdot v_{I_{t}} \mid \sigma=\left(I_{1} \leqslant I_{2} \leqslant \cdots \leqslant I_{t} \in S\right)\right\} .
$$

Lemma 3.5. The elements $\left[v_{I}\right]=v_{I}+\Theta$ span the $\mathbb{k}$-module $\mathbb{k}[S] / \Theta$.

Proof. Take any element $P_{\sigma}$ with $|\sigma| \geqslant 2$. Using relations in the face ring, we can express $P_{\sigma}=v_{I_{1}} \cdot \ldots \cdot v_{I_{t}}$ as $v_{i} \cdot v_{I_{1} \backslash i} \cdot \ldots \cdot v_{I_{t}}$ for a vertex $i \leqslant I_{1}$ by the following reason. Let $J \in i \vee\left(I_{1} \backslash i\right), J \neq I$. Suppose that $J \vee I_{2} \neq \varnothing$. Then a simplex $\widetilde{I} \in$ $J \vee I_{2}$ would contain two simplices $I_{1}$ and $J$ having the same set of vertices. This contradicts to the fact that $S_{\leqslant \widetilde{I}}$ is a boolean lattice. Therefore, we have $J \vee I_{2}=\varnothing$ and $v_{J} \cdot v_{I_{2}}=0$ for any $J \in i \vee\left(I_{1} \backslash i\right), J \neq I$. Thus $v_{I_{1}} \cdot v_{I_{2}}=v_{i} \cdot v_{I_{1} \backslash i} \cdot v_{I_{2}}$ from the relations in the face ring.

The element $v_{i}$ can be expressed as $\sum_{i^{\prime} \not I_{t}} a_{i^{\prime}} v_{i^{\prime}}$ modulo $\Theta$ according to (4) (we exclude all $v_{i}$ corresponding to the vertices of some maximal simplex $J \geqslant I_{t}$ ). Thus

$v_{i} v_{I_{t}}$ is expressed as a combination of $v_{I_{t}^{\prime}}$ with $I_{t}^{\prime}>1$ the element $P_{\sigma}$ is expressed as a linear combination of elements $P_{\sigma^{\prime}}$ that have either smaller length $t$ (in case $\left|I_{1}\right|=1$ ) or smaller $I_{1}$ (in case $\left|I_{1}\right|>1$ ). By iterating this descending process, we express the element $P_{\sigma}+\Theta \in \mathbb{k}[S] / \Theta$ as a linear combination of $\left[v_{I}\right]$.

\section{Linear relations on face classes}

Let $H_{T}^{*}(X)$ be a $T^{n}$-equivariant cohomology ring of $X$. Any proper face of $Q$ is acyclic, and therefore any face has a vertex. Hence, there exists a homomorphism $\mathbb{k}\left[S_{Q}\right] \hookrightarrow H_{T}^{*}(X)$, which sends the generator $v_{I}$ to the cohomology class, equivariant Poincare dual to $\left[X_{I}\right]$ (refer to $[\mathbf{9}$, Lm. 6.4] for details). The fiber inclusion in the Borel 
construction, $X \rightarrow X \times_{T} E T^{n}$, induces the ring homomorphism $H_{T}^{*}(X) \rightarrow H^{*}(X)$. The subspace of $H_{*}(X)$, Poincare dual to the image of the homomorphism

$$
g: \mathbb{k}\left[S_{Q}\right] \rightarrow H_{T}^{*}(X) \rightarrow H^{*}(X)
$$

is generated by the elements $\left[X_{I}\right]$, the fundamental classes of face submanifolds. Note that the elements $\left[X_{I}\right]=\left[F_{I}\right] \otimes \Omega_{I}$ can also be considered as the free generators of the $\mathbb{k}$-module

$$
\bigoplus_{q}\left(E_{X}\right)_{q, q}^{1}=\bigoplus_{q} \bigoplus_{|I|=n-q} H_{q}\left(F_{I}, \partial F_{I}\right) \otimes H_{q}\left(T^{n} / T_{I}\right) .
$$

Therefore, the submodule of $H_{*}(X)$ generated by $\left[X_{I}\right]$ coincides with the submodule $\bigoplus_{q}\left(E_{X}\right)_{q, q}^{\infty} \subset H_{*}(X)$. We call classes $\left[X_{I}\right]$ the face classes of $X$.

In the following let $\left\langle\left[X_{I}\right]\right\rangle$ denote the free $\mathbb{k}$-module generated by the elements $\left[X_{I}\right]$, $I \in S_{Q}$.

Proposition 4.1. Let $C_{I, A}$ be the constants defined in Lemma 3.3. The submodule of $H_{*}(X)$ generated by the face classes has linear relations of the following two types:

1. For each $J \in S,|J|=n-q-1$, and for each subset $A \subset[n],|A|=q$, there is a relation $R_{J, A}=0$, where

$$
R_{J, A}=\sum_{I, I>J}[I: J] C_{I, A}\left[X_{I}\right] .
$$

2. Let $q \leqslant n-2$, and let $\beta \in H_{q}(\partial Q)$ be a homology class lying in the image of the connecting homomorphism $\delta_{q+1}: H_{q+1}(Q, \partial Q) \rightarrow H_{q}(\partial Q)$. Let $\sum_{I,|I|=n-q} B_{I}\left[F_{I}\right]$ be a cellular chain representing $\beta$ (such representation exists since every face of $\partial Q$ is acyclic, thus may be considered as a homological cell). Then, for each subset $A \subset[n],|A|=q$, there is a relation $R_{\beta, A}^{\prime}=0$, where

$$
R_{\beta, A}^{\prime}=\sum_{I,|I|=n-q} B_{I} C_{I, A}\left[X_{I}\right] .
$$

Proof. The proof follows from the structure of homological spectral sequences of $X$ and $Y$. The module $\bigoplus_{q}\left(E_{X}\right)_{q, q}^{1}$ is freely generated by $\left[X_{I}\right]$. Relations on $\left[X_{I}\right]$ in $H_{*}(X)$ appear as the images of differentials landing at $\bigoplus_{q}\left(E_{X}\right)_{q, q}^{1}$. The relation $R_{J, A}$ is the image of the element

$$
\left[F_{J}\right] \otimes\left[T^{(A)}\right] \in H_{q+1}\left(F_{J}, \partial F_{J}\right) \otimes H_{q}\left(T^{n} / T_{J}\right) \subset\left(E_{X}\right)_{q+1, q}^{1}
$$

under the differential $\left(d_{X}\right)^{1}:\left(E_{X}\right)_{q+1, q}^{1} \rightarrow\left(E_{X}\right)_{q, q}^{1}$. Thus relations of the first type span the image of the first differentials hitting $\left(E_{X}\right)_{q, q}^{1}$.

Let us prove that images of higher differentials are generated by $R_{\beta, A}^{\prime}$. Higher differentials $\left(d_{Q}\right)^{\geqslant 2}$ coincide with $\delta_{*+1}: H_{*+1}(Q, \partial Q) \rightarrow H_{*}(\partial Q)$ by Proposition 2.5. The differentials $\left(d_{Y}\right) \geqslant 2$ coincide with $\delta_{*+1} \otimes \mathrm{id}_{\Lambda}$ by Proposition 2.6. Thus the image of $\left(d_{Y}\right) \geqslant 2$ in $\left(E_{Y}\right)_{q, q}^{2}$ is generated by the elements $\beta \otimes\left[T^{(A)}\right]$, which are, in turn, the homology classes of the elements

$$
\left(\sum_{|I|=n-q} B_{I}\left[F_{I}\right]\right) \otimes\left[T^{(A)}\right] \in\left(E_{Y}\right)_{q, q}^{1} .
$$

By Proposition 2.7, the differential $\left(d_{X}\right)^{r}$ landing at $\left(E_{X}\right)_{q, q}^{*}$ coincides with the composition of $\left(d_{Y}\right)^{r}$ and inclusion $f_{r}^{2}$. The map $f_{*}^{1}:\left(E_{Y}\right)_{q, q}^{1} \rightarrow\left(E_{X}\right)_{q, q}^{1}$ is the sum of the 
maps

$$
\mathrm{id} \otimes \varrho: H_{q}\left(F_{I}, \partial F_{I}\right) \otimes H_{q}\left(T^{n}\right) \rightarrow H_{q}\left(F_{I}, \partial F_{I}\right) \otimes H_{q}\left(T^{n} / T_{I}\right)
$$

over all simplices $I$ of rank $n-q$. Thus

$$
f_{*}^{r}\left(\beta \otimes\left[T^{(A)}\right]\right)=\left[f_{*}^{1}\left(\sum_{|I|=n-q} B_{I}\left[F_{I}\right] \otimes\left[T^{(A)}\right]\right)\right]=R_{\beta, A}^{\prime}
$$

by Lemma 3.3.

Remark 4.2. The element $R_{\beta, A}^{\prime} \in \bigoplus_{q}\left(E_{X}\right)_{q, q}^{2}$ does not depend on a cellular chain, representing $\beta$. Proposition 2.7 also implies that relations $\left\{R_{\beta, A}^{\prime}\right\}$ are linearly independent in $\left(E_{X}\right)_{q, q}^{2}$ when $\beta$ runs over some basis of $\operatorname{Im} \delta_{q+1}$ and $A$ runs over all subsets of $[n]$ of cardinality $q$.

Our next goal is to check that relations of the first type are exactly the relations in the quotient ring $\mathbb{k}\left[S_{Q}\right] / \Theta$.

Proposition 4.3. Let $\varphi:\left\langle\left[X_{I}\right]\right\rangle \rightarrow \mathbb{k}\left[S_{Q}\right]$ be the degree-reversing linear map, which sends the generator $\left[X_{I}\right]$ to $v_{I}$. Then $\varphi$ descends to the isomorphism of $\mathbb{k}$-modules

$$
\tilde{\varphi}:\left\langle\left[X_{I}\right]\right\rangle /\left\langle R_{J, A}\right\rangle \rightarrow \mathbb{k}\left[S_{Q}\right] / \Theta .
$$

Proof. (1) At first we prove that $\tilde{\varphi}$ is well defined by showing that the element

$$
\varphi\left(R_{J, A}\right)=\sum_{I, I>J}^{1}[I: J] C_{I, A} v_{I} \in \mathbb{k}\left[S_{Q}\right]
$$

lies in $\Theta$. Let $s=|J|$ and, consequently, $|I|=s+1,|A|=n-s-1$. Let $[n] \backslash A=$ $\left\{\alpha_{1}<\cdots<\alpha_{s+1}\right\}$, and let $\left\{j_{1}, \ldots, j_{s}\right\}$ be the vertices of $J$ listed in a positive order. Consider the $s \times(s+1)$ matrix

$$
\mathbf{D}=\left(\begin{array}{ccc}
\lambda_{j_{1}, \alpha_{1}} & \ldots & \lambda_{j_{1}, \alpha_{s+1}} \\
\vdots & \ddots & \vdots \\
\lambda_{j_{s}, \alpha_{1}} & \ldots & \lambda_{j_{s}, \alpha_{s+1}}
\end{array}\right)
$$

Denote by $\mathbf{D}_{l}$ the square submatrix obtained from $\mathbf{D}$ by deleting the $l$ th column, and let $a_{l}=(-1)^{l+1} \operatorname{det} \mathbf{D}_{l}$. We claim that

$$
\varphi\left(R_{J, A}\right)= \pm v_{J} \cdot\left(a_{1} \theta_{\alpha_{1}}+\cdots+a_{s+1} \theta_{\alpha_{s+1}}\right) \in \Theta
$$

Indeed, after expanding each $\theta_{l}$ as $\sum_{i \in \operatorname{Vert}(S)} \lambda_{i, l} v_{i}$, all elements of the form $v_{J} v_{i}$ with $i<J$ cancel (the coefficients of these terms are determinants of matrices with two coinciding rows). Other terms give

$$
\sum_{I>J, i=I \backslash J}\left(a_{1} \lambda_{i, \alpha_{1}}+\cdots+a_{s+1} \lambda_{i, \alpha_{s+1}}\right) v_{I} .
$$

The coefficient of $v_{I}$ is equal to

$$
\operatorname{det}\left(\begin{array}{ccc}
\lambda_{i, \alpha_{1}} & \cdots & \lambda_{i, \alpha_{s+1}} \\
\lambda_{j_{1}, \alpha_{1}} & \cdots & \lambda_{j_{1}, \alpha_{s+1}} \\
\vdots & \ddots & \vdots \\
\lambda_{j_{s}, \alpha_{1}} & \cdots & \lambda_{j_{s}, \alpha_{s+1}}
\end{array}\right)
$$

by the cofactor expansion along the first row. This determinant is equal to 
$\operatorname{sgn}_{A}[I: J] C_{I, A}$ since the ordering $i<j_{1}<\cdots<j_{s}$ of rows is either positive or negative depending on the incidence sign $[I: J]$.

(2) $\tilde{\varphi}$ is surjective by Lemma 3.5 .

(3) Ranks of both spaces are equal. Indeed, $\operatorname{dim}\left\langle\left[X_{I}\right]|| I \mid=n-q\right\rangle /\left\langle R_{J, A}\right\rangle=$ $\operatorname{dim}\left(E_{X}\right)_{q, q}^{2}=h_{n-q}^{\prime}\left(S_{Q}\right)$ by Proposition 2.7. By Proposition 2.4, the poset $S_{Q}$ is Buchsbaum. Thus $\operatorname{dim}\left(\mathbb{k}\left[S_{Q}\right] / \Theta\right)_{n-q}=h_{n-q}^{\prime}\left(S_{Q}\right)$ according to Schenzel's theorem ([14], [11, Prop.6.3]).

(4) If $\mathbb{k}$ is a field, the statement is proved. Let us denote the map $\widetilde{\varphi}$ defined over $\mathbb{k}$ by $\widetilde{\varphi}_{\mathbb{k}}$. The points $(1),(2)$ work over $\mathbb{Z}$, and thus $\widetilde{\varphi}_{\mathbb{Z}}$ is well defined and surjective. For any field $\mathbb{k}$ the functor $\otimes \mathbb{k}$ is right exact; hence $\widetilde{\varphi}_{\mathbb{k}}=\widetilde{\varphi}_{\mathbb{Z}} \otimes \mathbb{k}$. Since $\widetilde{\varphi}_{\mathbb{Z}} \otimes \mathbb{Q}$ is an isomorphism, $\operatorname{Ker} \widetilde{\varphi}_{\mathbb{Z}}$ is a torsion subgroup. If $\operatorname{Ker} \widetilde{\varphi}_{\mathbb{Z}}$ contains a summand $\mathbb{Z} / r \mathbb{Z} \neq 0$, then tensoring with $\mathbb{F}_{p}$, where $p$ is a prime factor of $r$, implies that $\operatorname{Ker} \widetilde{\varphi}_{\mathbb{Z}} \otimes \mathbb{F}_{p} \neq 0$ is the kernel of $\widetilde{\varphi}_{\mathbb{F}_{p}}$, which gives a contraction. Thus Ker $\widetilde{\varphi}_{\mathbb{Z}}=0$.

The Poincare duality on $X$ implies the following.

Corollary 4.4. The map $g: \mathbb{k}\left[S_{Q}\right] \rightarrow H^{*}(X)$ factors through $\mathbb{k}\left[S_{Q}\right] / \Theta$ and the kernel of the homomorphism $\tilde{g}: \mathbb{k}\left[S_{Q}\right] / \Theta \rightarrow H^{*}(X)$ is additively generated by the elements

$$
L_{\beta, A}^{\prime}=\sum_{I,|I|=n-q} B_{I} C_{I, A} v_{I},
$$

where $q \leqslant n-2, \beta \in \operatorname{Im}\left(\delta_{q+1}: H_{q+1}(Q, \partial Q) \rightarrow H_{q}(\partial Q)\right)$ is a homology class represented by a cellular chain $\sum_{I,|I|=n-q} B_{I}\left[F_{I}\right]$ in $\partial Q$ and $A \subset[n],|A|=q$.

Remark 4.5. The ideal $\Theta \subset \mathbb{k}\left[S_{Q}\right]$ coincides with the image of the homomorphism $H^{+}\left(B T^{n}\right) \rightarrow H_{T}^{*}(X)$. So the fact that $\Theta$ vanishes in $H^{*}(X)$ is not surprising. But we want to emphasize that $\Theta$ vanishes already at the second term of the spectral sequence, while other relations in $H^{*}(X)$ demonstrate the effects of higher differentials.

Note that the elements $R_{\beta, A}^{\prime}=\sum_{I,|I|=n-q} B_{I} C_{I, A}\left[X_{I}\right] \in\left(E_{X}\right)_{q, q}^{2}$ and

$$
L_{\beta, A}^{\prime}=\sum_{I,|I|=n-q} B_{I} C_{I, A} v_{I} \in \mathbb{k}\left[S_{Q}\right] / \Theta
$$

can be defined for any homology class $\beta \in H_{q}(\partial Q)$ (not only for the image of the connecting homomorphism).

Theorem 4.6. For every $\beta \in H_{q}(\partial Q), q \leqslant n-1$ and $A \subset[n],|A|=q$, the element $L_{\beta, A}^{\prime} \in \mathbb{k}\left[S_{Q}\right] / \Theta$ lies in a socle of $\mathbb{k}\left[S_{Q}\right] / \Theta$.

Recall that the socle of a module $\mathcal{M}$ over the polynomial ring $\mathbb{k}[m]$ is the submodule

$$
\operatorname{Soc} \mathcal{M} \stackrel{\text { def }}{=}\left\{y \in \mathcal{M} \mid \mathbb{k}[m]^{+} \cdot y=0\right\},
$$

where $\mathbb{k}[m]^{+}$is the maximal graded ideal of $\mathbb{k}[m]$.

We postpone the proof of Theorem 4.6 to Section 8 . 


\section{Non-face classes of $X$}

\subsection{Spine and diaphragm classes}

In this section we give a geometrical description of homology classes of $Q$ different from face classes.

Construction 5.1. Let $\eta \in H_{k}(Q)$ be a homology class of $Q$, and let $a \in H_{l}\left(T^{n}\right), l<k$, be a homology class of $T^{n}$ represented by a subtorus $T^{(a)} \subset T^{n}$. They determine the homology class $\eta \otimes a \in H_{k, l}(Y) \cong H_{k}(Q) \otimes H_{l}\left(T^{n}\right)$. Thus they determine the class $\operatorname{Sp}_{\eta, a}=f_{*}\left(\eta \otimes\left[T^{(a)}\right]\right) \in H_{k+l}(X)$ via the isomorphism $f_{*}: H_{k, l}(Y) \rightarrow H_{k, l}(X)$ asserted by point (1) of Proposition 2.9. The classes of this form will be called spine classes. If $\eta$ is represented by an embedded pseudomanifold $N \subset Q$ (we may assume that $N$ lies in the interior of $Q$ ), then the spine class $\operatorname{Sp}_{\eta, a}$ is represented by the pseudomanifold $N \times T^{(a)} \subset X$.

Construction 5.2. Let $\zeta \in H_{k}(Q, \partial Q)$ be a relative homology class for $k<n$. Suppose that $\zeta$ is represented by embedded pseudomanifold $L \subset Q$ of dimension $k$ with the boundary $\partial L \subset \partial Q$ (the boundary $\partial L$ may be empty). Every proper face of $Q$ is acyclic, and thus may be considered as a homological cell of $\partial Q$. Therefore, we may assume that $\partial L \subset Q_{k-1}$. We also assume that $L \backslash \partial L \subset Q \backslash \partial Q$. For each class $a \in$ $H_{l}\left(T^{n}\right)$, represented by an $l$-dimensional subtorus $T^{(a)}$, consider the subset $Z_{L, a}=$ $\left(L \times T^{(a)}\right) / \sim$ of the manifold $X$.

Proposition 5.3. 1. If $l \geqslant k$, the subset $Z_{L, a}$ is a pseudomanifold. Thus it represents a well-defined element $\operatorname{Df}_{L, a}=\left[Z_{L, a}\right] \in H_{k+l}(X)$, which will be called a diaphragm class.

2. If $l>k$, the class $\operatorname{Df}_{L, a} \in H_{k+l}(X)$ depends only on the class $\zeta=[L] \in H_{k}(Q, \partial Q)$ but not on its representative $L$.

Proof. The set $\left((L \backslash \partial L) \times T^{(a)}\right) / \sim=(L \backslash \partial L) \times T^{(a)}$ is a pseudomanifold of dimension $k+l$. The exceptional locus $\left(\partial L \times T^{(a)}\right) / \sim$ has dimension at most $k+l-2$. Indeed, we have $\partial L \subset Q_{k-1}$; thus, under the projection map $\left(\partial L \times T^{(a)}\right) / \sim \rightarrow \partial L$, every point $x \in \partial L$ has a preimage of the form $T^{(a)} / T_{I}$ with $|I| \geqslant n-k+1$. This set has dimension at most $l-1$ since $l+(n-k+1)>n$ (recall that $l \geqslant k$ by assumption). Thus the total dimension of exceptional locus is at most $\operatorname{dim} \partial L+(l-1)=$ $k+l-2$.

The second statement can be proved similarly. Let $\left(L_{1}, \partial L_{1}\right)$ and $\left(L_{2}, \partial L_{2}\right)$ be two pseudomanifolds representing the same element $\zeta \in H_{k}(Q, \partial Q)$. There exists a pseudomanifold bordism between them - that is, a pseudomanifold with boundary $\Xi$, $\operatorname{dim} \Xi=k+1$ and a map $\phi: \Xi \rightarrow Q$ such that $L_{1}, L_{2}$ are disjoint submanifolds of $\partial \Xi$, the restriction of $\phi$ to $L_{\epsilon}$ is the inclusion $L_{\epsilon} \hookrightarrow Q$, and $\phi\left(\partial \Xi \backslash\left(L_{1}^{\circ} \sqcup L_{2}^{\circ}\right)\right) \subset \partial Q$ (this follows from the geometrical definition of homology; see [13, App. A.2]). By acyclicity of proper faces we may again assume that $\phi\left(\partial \Xi \backslash\left(L_{1}^{\circ} \sqcup L_{2}^{\circ}\right)\right)$ lies in the stratum $Q_{k}$. Similar to the first statement, we can consider the space $\left(\Xi \times T^{(a)}\right) / \sim$ of dimension $k+l+1$. This space is a pseudomanifold with boundary, and the boundary is exactly the difference $Z_{L_{1}, a}-Z_{L_{2}, a}$. Thus $\operatorname{Df}_{L_{1}, a}=\operatorname{Df}_{L_{2}, a}$ in $H_{*}(X)$.

Thus for $k<l$ there is a well-defined homology class $\operatorname{Df}_{\zeta, a} \stackrel{\text { def }}{=} \operatorname{Df}_{L, a} \in H_{k, l}(X)$ depending on $\zeta \in H_{k}(Q, \partial Q)$ and $a \in H_{l}\left(T^{n}\right)$. These classes span the homology modules $H_{k, l}(X)$ for $k<l$ and correspond to point (2) of Proposition 2.9. 
When $k=l<n$ we call the classes $\operatorname{Df}_{L, a}$ extremal diaphragm classes. In this case the situation is different: the classes $\operatorname{Df}_{L, a}$ depend not only on the homology class of $L$ but on the representative $L$ itself. Nevertheless, if $L_{1}$ and $L_{2}$ represent the same class in $H_{k}(Q, \partial Q)$, then the classes $\mathrm{Df}_{L_{1}, a}, \mathrm{Df}_{L_{2}, a} \in H_{k, k}(X)$ coincide modulo face classes, as proved below. Our goal is to derive exact formulas; thus we restrict to the case when homology class $a \in H_{l}\left(T^{n}\right)$ is represented by a coordinate subtorus $T^{(A)}$ for $A \subset[n],|A|=l$.

Construction 5.4. Let $\phi_{\epsilon}:\left(L_{\epsilon}, \partial L_{\epsilon}\right) \rightarrow(Q, \partial Q), \epsilon=1,2$, be two pseudomanifolds representing the same element $\zeta \in H_{k}(Q, \partial Q), k<n$. As in the proof of Proposition 5.3, consider a pseudomanifold bordism $(\Xi, \partial \Xi)$ between $L_{1}$ and $L_{2}$, and the map $\phi: \Xi \rightarrow$ $Q$, which sends the boundary $\partial L$ into the union of $L_{1}, L_{2}$, and $Q_{k}$. The skeletal stratification of $Q$ induces a stratification on $\Xi$. The restriction of the map $\phi$ sends $\Xi_{k-1}$ to $Q_{k-1}$. Let $\delta$ be the connecting homomorphism $\delta: H_{k+1}(\Xi, \partial \Xi) \rightarrow H_{k}\left(\partial \Xi, \Xi_{k-1}\right)$ in the long exact sequence of the triple $\left(\Xi, \partial \Xi, \Xi_{k-1}\right)$. The composite homomorphism

$$
\begin{aligned}
& H_{k+1}(\Xi, \partial \Xi) \stackrel{\delta}{\rightarrow} H_{k}\left(\partial \Xi, \Xi_{k-1}\right) \cong \\
& H_{k}\left(L_{1}, \partial L_{1}\right) \oplus H_{k}\left(L_{2}, \partial L_{2}\right) \oplus H_{k}\left(\partial \Xi \backslash\left(L_{1}^{\circ} \cup L_{2}^{\circ}\right), \partial \Xi_{k-1}\right) \stackrel{\operatorname{id} \oplus \operatorname{id} \oplus \phi_{*}}{\longrightarrow} \\
& H_{k}\left(L_{1}, \partial L_{1}\right) \oplus H_{k}\left(L_{2}, \partial L_{2}\right) \oplus H_{k}\left(Q_{k}, Q_{k-1}\right)
\end{aligned}
$$

maps the fundamental class $[\Xi] \in H_{k+1}(\Xi, \partial \Xi)$ to the element

$$
\left(\left[L_{1}\right],-\left[L_{2}\right], \sum_{I, \operatorname{dim} F_{I}=k} D_{I}\left[F_{I}\right]\right)
$$

for some coefficients $D_{I} \in \mathbb{k}$.

Proposition 5.5. Let $L_{1}, L_{2}$ be two pseudomanifolds representing the same class $\zeta \in$ $H_{k}(Q, \partial Q), k<n$. Consider any subset $A \subset[n],|A|=k$, and let $a \in H_{k}\left(T^{n}\right)$ be the fundamental class of the coordinate subtorus $T^{(A)}$. Then there is a relation in $H_{2 k}(X)$ :

$$
\operatorname{Df}_{L_{1}, a}-\operatorname{Df}_{L_{2}, a}+\sum_{I, \operatorname{dim} F_{I}=k} D_{I} C_{I, A}\left[X_{I}\right]=0
$$

The numbers $D_{I}$ are given by (5), and the numbers $C_{I, A}$ were defined in Lemma 3.3.

Proof. Consider the space $\left(\Xi \times T^{(A)}\right) / \approx$ and the map $\phi \times \iota:\left(\Xi \times T^{(A)}\right) / \approx \rightarrow X=$ $\left(Q \times T^{n}\right) / \sim$, where the relation $\approx$ is induced from $\sim$ by the map $\phi$, and $\iota: T^{(A)} \rightarrow T^{n}$ is the inclusion map. The space $\left(\Xi \times T^{(A)}\right) / \approx$ is a pseudomanifold with boundary, and its boundary represents the element (6) in homology.

Therefore, up to face classes, the middle homology group $H_{k, k}(X)$ coincides with $H_{k}(Q, \partial Q) \otimes H_{k}\left(T^{n}\right)$ for $k<n$. This is exactly the statement of Proposition 2.9, point (3). 


\subsection{Integral coefficients}

Proposition 2.9 was stated only over a field. On the other hand, the geometrical constructions of the previous subsection yield the additive homomorphisms over $\mathbb{Z}$

$$
\bigoplus_{k>l} H_{k}(Q) \otimes H_{l}\left(T^{n}\right) \rightarrow H_{*}(X), \quad \bigoplus_{k<l} H_{k}(Q, \partial Q) \otimes H_{l}\left(T^{n}\right) \rightarrow H_{*}(X) .
$$

Let $H_{k, l}(X ; \mathbb{Z}) \subset H_{*}(X ; \mathbb{Z})$ denote the image of the group $H_{k}(Q ; \mathbb{Z}) \otimes H_{l}\left(T^{n} ; \mathbb{Z}\right)$ when $k>l$, or the image of the group $H_{k}(Q, \partial Q ; \mathbb{Z}) \otimes H_{l}\left(T^{n} ; \mathbb{Z}\right)$ when $k<l$, and let $H_{k, k}(X ; \mathbb{Z})$ denote the the subgroup of $H_{*}(X ; \mathbb{Z})$ generated by all extremal diaphragm classes and all face classes. Then we have a decomposition $H_{*}(X ; \mathbb{Z}) \cong \bigoplus_{k, l} H_{k, l}(X ; \mathbb{Z})$ over integers, since it holds over any field. This proves the following proposition.

Proposition 5.6. Proposition 2.9 holds over $\mathbb{Z}$. Homology groups of $X$ are generated by face classes, spine classes, and diaphragm classes. The groups $H_{k, l}(X ; \mathbb{Z})$ for $k>l$ are generated by spine classes; the groups $H_{k, l}(X ; \mathbb{Z})$ for $k<l$ are generated by nonextremal diaphragm classes; the short exact sequence

$$
0 \rightarrow\left(\dot{E}_{X}\right)_{k, k}^{\infty} \rightarrow H_{k, k}(X ; \mathbb{Z}) \rightarrow H_{k}(Q, \partial Q ; \mathbb{Z}) \otimes H_{k}\left(T^{n} ; \mathbb{Z}\right) \rightarrow 0
$$

identifies the quotient of $H_{k, k}(X ; \mathbb{Z})$ by the face classes with the group of extremal diaphragm classes when $k<n$.

\section{Intersections in $H_{*}(X)$}

Remark 6.1. In construction 5.1 we defined the classes $\operatorname{Sp}_{\eta, a}$ for each $\eta \in H_{k}(Q)$ and $a \in H_{l}\left(T^{n}\right)$ under the assumption $k>l$. The same construction can be applied for any $k$ and $l$. If $\eta$ is represented by a pseudomanifold $N$ lying in the interior of $Q$, and $a$ is represented by a subtorus, then the product $N \times T^{(a)}$ is a pseudomanifold in $X$, and thus represents an element $\left[N \times T^{(a)}\right] \in H_{k+l}(X)$. Although for $k \leqslant l$ this element is not a spine class, we keep denoting it $\operatorname{Sp}_{\eta, a}$. By construction, if $k \leqslant l$, the class $\mathrm{Sp}_{\eta, a}$ coincides with the diaphragm class $\mathrm{Df}_{N, a}$, and in particular if $k<l$, there holds $\mathrm{Sp}_{\eta, a}=\mathrm{Df}_{\eta^{\prime}, a}$, where $\eta^{\prime}$ is the image of $\eta$ in $H_{k}(Q, \partial Q)$.

Let $\cap: H_{k}(M) \otimes H_{l}(M) \rightarrow H_{k+l-\operatorname{dim} M}(M)$ denote the intersection product on a closed manifold $M$, the operation that is Poincare dual to the cup-product in cohomology. From the geometrical structure of face classes (and also from Corollary 4.4) follows:

Proposition 6.2. If $I_{1}, I_{2} \in S_{Q}$, then $\left[X_{I_{1}}\right] \cap\left[X_{I_{2}}\right]=\left[X_{I_{1} \cap I_{2}}\right] \cap \sum_{J \in I_{1} \vee I_{2}}\left[X_{J}\right]$.

Intersections of spine and diaphragm classes can also be described geometrically. There are intersection products $\cap: H_{k_{1}}(Q) \otimes H_{k_{2}}(Q, \partial Q) \rightarrow H_{k_{1}+k_{2}-n}(Q)$ and $\cap: H_{l_{1}}\left(T^{n}\right) \otimes H_{l_{2}}\left(T^{n}\right) \rightarrow H_{l_{1}+l_{2}-n}\left(T^{n}\right)$. By the construction of homology classes in $X$ we have the following properties of the intersection product on $H_{*}(X)$.

Proposition 6.3. 1. The classes $\operatorname{Sp}_{\eta, a} \in H_{k_{1}, l_{1}}(X), k_{1}>l_{1}$, and $\mathrm{Df}_{L, b} \in H_{k_{2}, l_{2}}(X)$, $k_{2} \leqslant l_{2}$, satisfy

$$
\mathrm{Sp}_{\eta, a} \cap \mathrm{Df}_{L, b}=\mathrm{Sp}_{\eta \cap[L], a \cap b} .
$$

Since $\operatorname{dim}(\eta \cap[L])=k_{1}+k_{2}-n$ and $\operatorname{dim}(a \cap b)=l_{1}+l_{2}-n$, the element $\mathrm{Sp}_{\eta \cap[L], a \cap b}$ is either a spine class (if $k_{1}+k_{2}>l_{1}+l_{2}$ ) or a diaphragm class conventionally defined in Remark 6.1 (if $k_{1}+k_{2} \leqslant l_{1}+l_{2}$ ). 
2. The classes $\operatorname{Sp}_{\eta_{1}, a_{1}} \in H_{k_{1}, l_{1}}(X), k_{1}>l_{1}$, and $\mathrm{Sp}_{\eta_{2}, a_{2}} \in H_{k_{2}, l_{2}}(X), k_{2}>l_{2}$, satisfy

$$
\mathrm{Sp}_{\eta_{1}, a_{1}} \cap \mathrm{Sp}_{\eta_{2}, a_{2}}=\mathrm{Sp}_{\eta_{1} \cap \eta_{2}, a_{1} \cap a_{2}} \text {. }
$$

The result is a spine class.

3. Spine classes do not meet face classes: $\mathrm{Sp}_{\eta, a} \cap\left[X_{I}\right]=0$.

Proposition 6.4. The linear span of proper face classes $\left[X_{I}\right]$ is an ideal of $H_{*}(X)$ with respect to the intersection product.

Proof. Suppose $I \neq \hat{0}$, and let $\imath: X_{I} \hookrightarrow X$ be the inclusion of a face submanifold. Let $\kappa$ be a cohomology class Poincare dual to a diaphragm class Df in $X$. Then $\left[X_{I}\right] \cap \mathrm{Df}=\left[X_{I}\right] \frown \kappa=\imath_{*}\left(\imath^{*}(\kappa) \frown\left[X_{I}\right]\right)$. Since $X_{I}$ is a manifold with locally standard action whose orbit space is acyclic together with all faces, the face classes of $X_{I}$ span its homology. Hence the class $\imath^{*}(\kappa) \frown\left[X_{I}\right] \in H_{*}\left(X_{I}\right)$ is a linear combination of face classes in $H_{*}\left(X_{I}\right)$ and therefore $\imath_{*}\left(\imath^{*}(\kappa) \frown\left[X_{I}\right]\right)$ is a linear combination of face classes in $H_{*}(X)$.

Remark 6.5. Intersections of diaphragm classes with themselves and with face classes are more difficult to describe in general. Nevertheless, in practice one can use the following trick (cf. the discussion of a similar problem in [3, Sect. 8]). Suppose the task is to compute $\operatorname{Df}_{L, a} \cap\left[X_{I}\right]$. If $L \cap F_{I}=\varnothing$, then the intersection product is 0 . If not, find another representative $L^{\prime}$ such that $\left[L^{\prime}\right]=[L] \in H_{*}(Q, \partial Q)$ and $L^{\prime} \cap F_{I}=\varnothing$. Then, by Proposition 5.5, $\mathrm{Df}_{L, a}=\mathrm{Df}_{L^{\prime}, a}+\Sigma$, where $\Sigma$ is a linear combination of face classes. Thus we have $\operatorname{Df}_{L, a} \cap\left[X_{I}\right]=\operatorname{Df}_{L^{\prime}, a} \cap\left[X_{I}\right]+\Sigma \cap\left[X_{I}\right]=\Sigma \cap\left[X_{I}\right]$, which can be computed by Proposition 6.2.

\section{Examples}

\subsection{One concrete example}

It is similar to the one studied by Poddar and Sarkar in [12, Th. 3.1]. Let $Q$ be a square with triangular hole. Orientations of facets and values of characteristic function are assigned to $Q$ as shown in Figure 1 (left). Homology groups of the corresponding 4-dimensional manifold $X=\left(Q \times T^{2}\right) / \sim$ are described below.
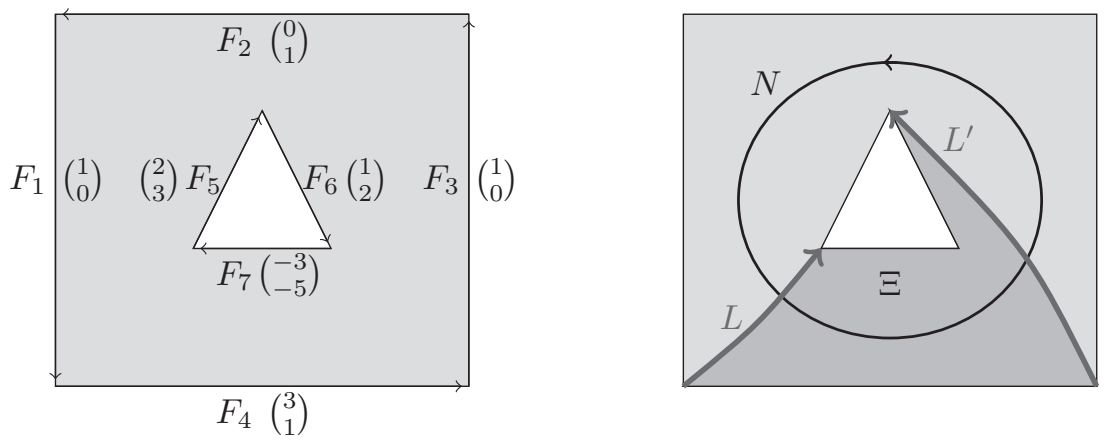

Figure 1: Structure of $Q$ and values of characteristic function 
(1) Face classes. These are the following: the fundamental class $[X] \in H_{4}(X)$; the classes of characteristic submanifolds $\left[X_{1}\right],\left[X_{2}\right], \ldots,\left[X_{7}\right] \in H_{1,1}(X) \subset H_{2}(X)$, which correspond to the sides of $Q$; and the classes of fixed points of the action

$$
\left[X_{12}\right],\left[X_{23}\right],\left[X_{34}\right],\left[X_{14}\right],\left[X_{56}\right],\left[X_{67}\right],\left[X_{57}\right] \in H_{0,0}(X)=H_{0}(X),
$$

which correspond to the vertices of $Q$. Relations on these classes are given by Proposition 4.1. The first-type relations in $H_{2}(X)$ are

$$
\begin{gathered}
{\left[X_{1}\right]+\left[X_{3}\right]+3\left[X_{4}\right]+2\left[X_{5}\right]+\left[X_{6}\right]-3\left[X_{7}\right]=0,} \\
{\left[X_{2}\right]+\left[X_{4}\right]+3\left[X_{5}\right]+2\left[X_{6}\right]-5\left[X_{7}\right]=0}
\end{gathered}
$$

(the coefficients are, respectively, first and second coordinates of the values of characteristic function). The first type relations on the classes $\left[X_{i j}\right]$ are encoded by the sides of of the orbit space. These relations are the following:

$$
\left[X_{12}\right]=-\left[X_{23}\right]=\left[X_{34}\right]=-\left[X_{14}\right], \quad\left[X_{56}\right]=\left[X_{67}\right]=\left[X_{57}\right] .
$$

To find relations of the second type, we need to pick a homology class in the image of $\delta_{1}: H_{1}(Q, \partial Q) \rightarrow H_{0}(\partial Q)$. Take, for example, the class, represented by the chain $\left[F_{14}\right]-\left[F_{57}\right]$. It induces the relation of second type:

$$
\left[X_{14}\right]-\left[X_{57}\right]=0 .
$$

Thus all fixed points represent the same up to sign generator $[\mathrm{pt}] \in H_{0}(X)$. Certainly, this easily follows from the connectivity of $X$, but we wanted to emphasize the different nature of two types of relations.

(2) Spine classes. Consider a submanifold $N \subset Q$ representing the generator $\eta \in$ $H_{1}(Q)$ (Figure 1, right). Together with the class of a point $\left[T^{(\varnothing)}\right] \in H_{0}\left(T^{2}\right)$ it determines a spine class $\mathrm{Sp}_{\eta, \varnothing} \in H_{1,0}(X)=H_{1}(X)$. Geometrically, $\mathrm{Sp}_{\eta, \varnothing}$ is represented by a submanifold $N \subset Q$ lifted by a zero-section map $Q \hookrightarrow X$.

(3) Diaphragm classes. Consider a submanifold $L$ representing the generator of $H_{1}(Q, \partial Q)$ and assume that $\partial L$ lies in the 0 -skeleton of $Q$ (Figure 1, right). For each subset $A=\{1\},\{2\},\{1,2\}$ we have a homology class in $H_{1,|A|}(X)$ represented by a pseudomanifold $\left(L \times T^{(A)}\right) / \sim$. Thus we have the generators

$$
\operatorname{Df}_{L, 1}=\left[\left(L \times T^{(\{1\})}\right) / \sim\right], \quad \operatorname{Df}_{L, 2}=\left[\left(L \times T^{(\{2\})}\right) / \sim\right]
$$

of $H_{1,1}(X) \subset H_{2}(X)$ and the generator $\operatorname{Df}_{L,\{12\}}=\left[\left(L \times T^{2}\right) / \sim\right]$ of $H_{1,2}(X)=H_{3}(X)$. Let $L^{\prime}$ be another submanifold representing the same homology class in $H_{1}(Q, \partial Q)$. Consider a bordism $\Xi$ between $L$ and $L^{\prime}$ shown on the figure. We have $\operatorname{Df}_{L,\{12\}}=$ $\mathrm{Df}_{L^{\prime},\{12\}}$ in $H_{3}(X)$ since $\left(\Xi \times T^{2}\right) / \sim$ is a pseudomanifold bordism between $(L \times$ $\left.T^{2}\right) / \sim$ and $\left(L^{\prime} \times T^{2}\right) / \sim$.

We have a relation $\delta \Xi=-[L]+\left[L^{\prime}\right]+\left[F_{4}\right]+\left[F_{6}\right]+\left[F_{7}\right]$ that induces the relations

$$
\begin{aligned}
& -\mathrm{Df}_{L, 1}+\mathrm{Df}_{L^{\prime}, 1}+1\left[X_{4}\right]+2\left[X_{6}\right]-5\left[X_{7}\right]=0, \\
& -\mathrm{Df}_{L, 2}+\mathrm{Df}_{L^{\prime}, 2}+3\left[X_{4}\right]+1\left[X_{6}\right]-3\left[X_{7}\right]=0
\end{aligned}
$$

in $H_{2}(X)$. These relations are the boundaries of $\left(\Xi \times T^{(\{1\})}\right) / \sim$ and $\left(\Xi \times T^{(\{2\})}\right) / \sim$ respectively. The coefficients are the complimentary coordinates of the characteristic 
function: for the class encoded by the first coordinate subtorus, we take the second coordinate, and vice versa. In this computation we applied the formula for the coefficients $C_{I, A}$ asserted by Lemma 3.3 .

(4) Intersections of classes. It is easily seen that the classes $\mathrm{Sp}_{N, \varnothing}$ and $\operatorname{Df}_{L,\{1,2\}}$ are transversal, and their intersection induces a nondegenerate pairing between $H_{1}(X)$ and $H_{3}(X)$.

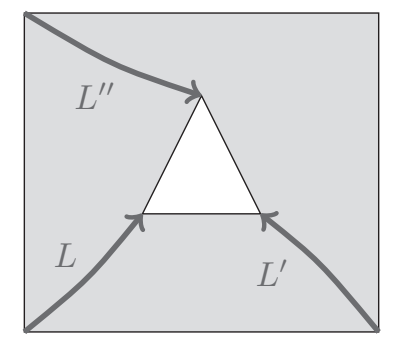

Figure 2: Different representatives of diaphragm classes

Let us compute the intersection of $\operatorname{Df}_{L, 1}$ with $\operatorname{Df}_{L, 2}$ to demonstrate the idea sketched in Remark 6.5. Consider the auxiliary intervals $L^{\prime}$ and $L^{\prime \prime}$ shown on Figure 2. Similar to the previous computations we get

$$
\operatorname{Df}_{L, 1}=\operatorname{Df}_{L^{\prime}, 1}+1\left[X_{4}\right]-5\left[X_{7}\right], \quad \operatorname{Df}_{L, 2}=\operatorname{Df}_{L^{\prime \prime}, 2}-1\left[X_{1}\right]-2\left[X_{5}\right] .
$$

Thus

$$
\begin{aligned}
\operatorname{Df}_{L, 1} \cap \mathrm{Df}_{L, 2} & =\left(\mathrm{Df}_{L^{\prime}, 1}+\left[X_{4}\right]-5\left[X_{7}\right]\right) \cap\left(\mathrm{Df}_{L^{\prime \prime}, 2}-\left[X_{1}\right]-2\left[X_{5}\right]\right) \\
& =-\left[X_{4}\right] \cap\left[X_{1}\right]+10\left[X_{7}\right] \cap\left[X_{5}\right]=-\left[X_{14}\right]+10\left[X_{57}\right]=9[\mathrm{pt}] \in H_{0}(X) .
\end{aligned}
$$

\subsection{Toric origami manifolds}

In this subsection we apply our method to the class of toric origami manifolds and show that some results of $[\mathbf{3}]$ can be derived from the spectral sequence of the orbit type filtration. The toric origami manifolds were introduced in differential geometry by Cannas da Silva, Guillemin, and Pires [7] as generalizations of symplectic toric manifolds. The precise geometrical definition is irrelevant to our study, but we review the essential topological properties. An orientable toric origami manifold $X$ is a manifold with locally standard torus action; its orbit space $Q=X / T^{n}$ is homotopy equivalent to a graph $\Gamma$, and the inclusion of any face in the orbit space is homotopy equivalent to an inclusion of some subgraph in $\Gamma$. As before, there is a principal torus bundle $Y \rightarrow Q$ such that $X=Y / \sim$. Since $Q$ is homotopy equivalent to a graph, $H^{2}\left(Q, \mathbb{Z}^{n}\right)=0$, so the Euler class of $Y$ vanishes. Thus in the origami case there always holds $X=Q \times T^{n} / \sim$.

Now we restrict to the case when all proper faces of $Q$ are acyclic. Since the faces are homotopy equivalent to graphs, they are contractible. Let $b_{1}=\operatorname{dim} H_{1}(Q)=$ $\operatorname{dim} H_{1}(\Gamma)$. The Poincare-Lefchetz duality implies

$$
H_{q}(Q, \partial Q ; \mathbb{Z}) \cong H^{n-q}(Q ; \mathbb{Z}) \cong\left\{\begin{array}{l}
\mathbb{Z}, \text { if } q=n ; \\
\mathbb{Z}^{b_{1}}, \text { if } q=n-1 ; \\
0, \text { otherwise. }
\end{array}\right.
$$


Let us describe the connecting homomorphisms $\delta_{i}: H_{i}(Q, \partial Q ; \mathbb{Z}) \rightarrow H_{i-1}(\partial Q ; \mathbb{Z})$. For simplicity we discuss the case $n \geqslant 4$; dimensions 2 and 3 can be done similarly. When $n \geqslant 4$, lacunas in the exact sequence of the pair $(Q, \partial Q)$ imply that the map $\delta_{i}: H_{i}(Q, \partial Q ; \mathbb{Z}) \rightarrow H_{i-1}(\partial Q ; \mathbb{Z})$ is an isomorphism for $i=n-1, n$, and trivial otherwise. Thus we have

$$
H_{i}(\partial Q ; \mathbb{Z}) \cong\left\{\begin{array}{l}
\mathbb{Z}, \text { if } i=0 \text { or } n-1 \\
\mathbb{Z}^{b_{1}}, \text { if } i=1 \text { or } n-2 \\
0, \text { otherwise. }
\end{array}\right.
$$

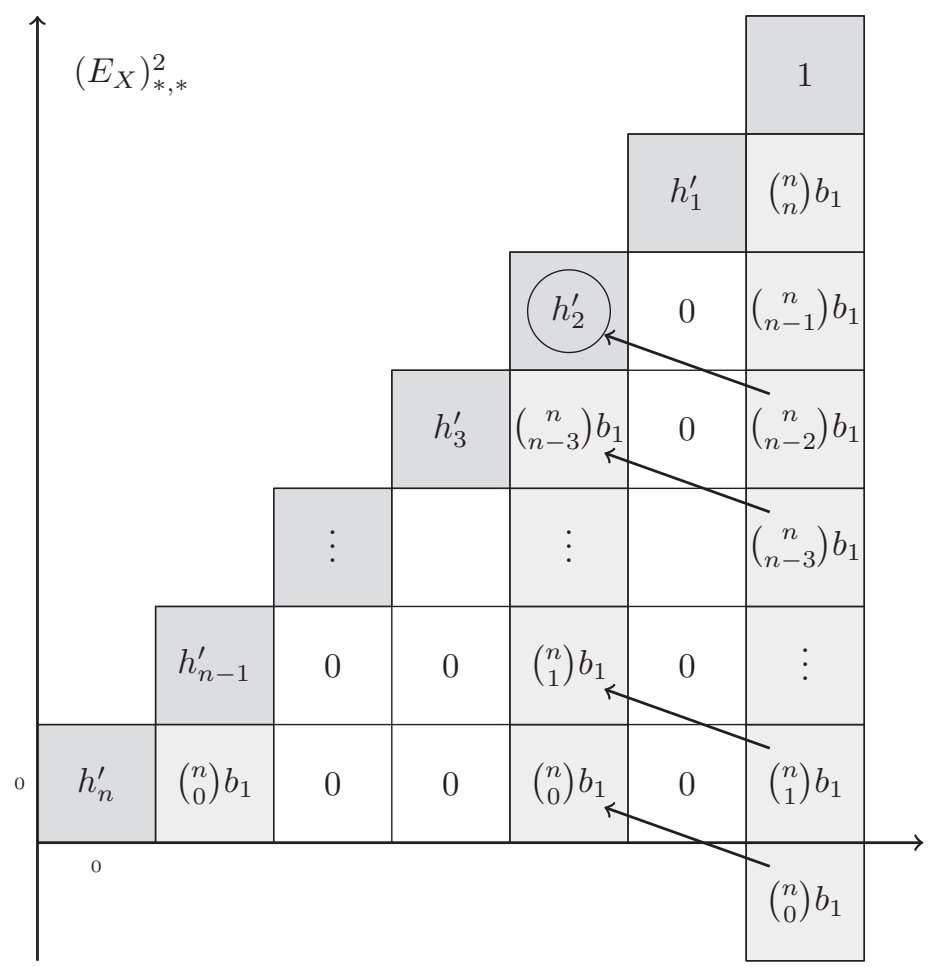

Figure 3: Homology spectral sequence of the orbit type filtration in the case of an orientable origami manifold with acyclic proper faces of the orbit space. Only the ranks of modules are written to save space.

Proposition 2.7 implies that $\left(E_{X}\right)_{p, q}^{2}$ has the form shown schematically in Figure 3 . The differential $\left(d_{X}\right)^{2}$ hitting the marked position produces relations $R_{\beta, A}^{\prime}$ of the second type on the classes $\left[X_{I}\right] \in H^{2 n-4}(X ; \mathbb{Z})$. These relations are explicitly described by Proposition 4.1, and the number of independent relations is $\left(\begin{array}{l}n \\ 2\end{array}\right) b_{1}$. Dually, this consideration shows that the map $\mathbb{Z}\left[S_{Q}\right] / \Theta \rightarrow H^{*}(X ; \mathbb{Z})$ has a nontrivial kernel of dimension $\left(\begin{array}{l}n \\ 2\end{array}\right) b_{1}$ in degree 4 .

In addition, there are non-face classes (nondiagonal terms in the spectral sequence) as follows: 
1. There are $b_{1}$ 1-dimensional spine classes, which are as the liftings of cycles in $\Gamma$.

2. There are $b_{1}$ diaphragm classes of codimension 1 given by the generators of $H_{n-1}(Q, \partial Q ; \mathbb{Z}) \cong H^{1}(\Gamma)$ swept by the action of the whole torus. These classes are equivariant. There is a nondegenerate intersection pairing between these classes and the spine classes.

3. There are $n b_{1}$ extremal diaphragm classes of codimension 2 . These are given by the generators of $H_{n-1}(Q, \partial Q ; \mathbb{Z})$ lifted to $X$ and swept by actions of $(n-1)$ dimensional subtori. The choice of these classes is not canonical.

\section{Collar model}

In this section we prove Theorem 4.6, stated in Section 4, using an auxiliary space $\widehat{X}$.

Construction 8.1. Consider the space $\widehat{Q}=\partial Q \times[0,1]$, which is an $(n-1)$-dimensional manifold with boundary. The boundary $\partial \widehat{Q}$ has the form $\partial_{0} \widehat{Q} \sqcup \partial_{1} \widehat{Q}$, where $\partial_{\epsilon} \widehat{Q}=$ $\partial Q \times\{\epsilon\}, \epsilon=0,1$. We may identify $\partial_{0} \widehat{Q}$ with $\partial Q$ and consider the filtration

$$
Q_{0} \subset Q_{1} \subset \cdots \subset Q_{n-1}=\partial Q=\partial_{0} \widehat{Q} \subset \widehat{Q} .
$$

The space $\widehat{Q}$ can be considered as a collar of $\partial Q$ inside $Q$.

Consider the space $\widehat{Y}=\widehat{Q} \times T^{n}$ and the identification space $\widehat{X}=\widehat{Y} / \sim$. The relation $\sim$ identifies points over $\partial_{0} \widehat{Q}$ as in Construction 2.2, while no identifications are imposed over $\partial_{1} \widehat{Q}$. The space $\widehat{X}$ is a manifold with boundary. Its boundary consists of points over $\partial_{1} Q$. The space $\widehat{X}$ can be considered as a $T^{n}$-invariant tubular neighborhood of the union of all characteristic submanifolds in $X$. There are natural topological filtrations on $\widehat{Y}$ and $\widehat{X}$ induced by the filtration on $\widehat{Q}$.

In the terminology of $[\mathbf{2}]$ the space $\widehat{X}$ is a Buchsbaum pseudo-cell complex, and thus Propositions 2.5 and 2.6 and items (1)-(5) of Proposition 2.7 hold for $\widehat{Q}, \widehat{Y}$, and $\widehat{X}$. The $n$th column of all spectral sequences vanishes since $H_{*}\left(\widehat{Q}, \partial_{0} \widehat{Q}\right)=0$. Thus the spectral sequences $\left(\dot{E}_{\widehat{Q}}\right)^{r},\left(\dot{E}_{\widehat{Y}}\right)^{r},\left(\dot{E}_{\widehat{X}}\right)^{r}$ collapse at first pages and, consequently, the spectral sequences $\left(E_{\widehat{Q}}\right)^{r},\left(E_{\widehat{Y}}\right)^{r},\left(E_{\widehat{X}}\right)^{r}$ collapse at second pages.

For each $I \in S_{Q}$, with $\operatorname{dim} F_{I}=q<n$ there is a distinguished element $\left[X_{I}\right] \in$ $H_{2 q}\left(\widehat{X}_{q}, \widehat{X}_{q-1}\right)=\left(E_{\widehat{X}}\right)_{q, q}^{1}$. It survives in the spectral sequence and represents the fundamental class of the face submanifold $X_{I} \subset \widehat{X}$. Linear relations on classes $\left[X_{I}\right]$ in $H_{*}(\widehat{X})$ are described as in Section 4 . When $q=n-1$, there are no relations on $\left[X_{I}\right]$ since there are no differentials landing at the cell $\left(E_{\widehat{X}}\right)_{n-1, n-1}^{1}$. For $q<n-1$ the relations on $\left[X_{I}\right]$ are the images of $\left(d_{\widehat{X}}\right)^{1}:\left(E_{\widehat{X}}\right)_{q+1, q}^{1} \rightarrow\left(E_{\widehat{X}}\right)_{q, q}^{1}$. These differentials coincide with $\left(d_{X}\right)^{1}$ and thus, for $|I|>1$, the relations on $\left[X_{I}\right]$ are exactly $R_{J, A}$, defined in Proposition 4.1. Hence Proposition 4.3 implies the following.

Lemma 8.2. Let $V_{*}$ denote the submodule of $H_{*}(\widehat{X})$ generated by the face classes $\left[X_{I}\right], I \neq \hat{0}$. Then there exists a degree-reversing linear map

$$
\tilde{\varphi}: V_{2 q} \rightarrow\left(\mathbb{k}\left[S_{Q}\right] / \Theta\right)_{2(n-q)}
$$

that sends $\left[X_{I}\right]$ to $v_{I}$. It is an isomorphism for $q<n-1$ and surjective for $q=n-1$. 
This map is a ring homomorphism with respect to the intersection product on $\widehat{X}$ and the product in the face ring.

Now we are in position to give a geometrical proof of Theorem 4.6.

Proof. We need to show that the element $L_{\beta, A}^{\prime}$ lies in a socle of $\mathbb{k}\left[S_{Q}\right] / \Theta$ - that is, $L_{\beta, A}^{\prime} \cdot v_{i}=0$ for any vertex $i \in \operatorname{Vert}\left(S_{Q}\right)$. By Lemma 8.2 it is enough to show that $R_{\beta, A}^{\prime} \cap\left[F_{i}\right]=0$ in $H_{*}(\widehat{X})$.

Consider a geometrical cycle $N \subset \partial_{0} \widehat{Q}$ representing $\beta$. Now we allow $N$ to be any representative, and do not require that it lies in the stratum $Q_{q}$. Consider the cycle $N \times T^{(A)}$ in $\partial_{0} \widehat{Q} \times T^{n}$, and the corresponding cycle $\left(N \times T^{(A)}\right) / \sim$ in $\widehat{X}$. The latter cycle represents the class $R_{\beta, A}^{\prime} \in H_{2 q}(\widehat{X})$ by definition. We can move the cycle $N \subset \widehat{Q}$ away from the boundary $\partial_{0} \widehat{Q}$. Thus $N \cap\left[F_{i}\right]=\varnothing$ and therefore $R_{\beta, A}^{\prime} \cap\left[X_{i}\right]=0$ in $H_{*}(\widehat{X})$.

Remark 8.3. The same argument proves that $L_{\beta, A}^{\prime} \cdot v_{I}=0$ for any simplex $I \in S_{Q} \backslash \hat{0}$. This fact does not directly follow from Theorem 4.6, since the map $\mathbb{k}[m] \rightarrow \mathbb{k}\left[S_{Q}\right] / \Theta$ may be nonsurjective in general.

Remark 8.4. The only reason why we considered the collar model $\widehat{X}$ instead of $X$ is that there are no additional relations in $H_{*}(\widehat{X})$ compared with $\mathbb{k}\left[S_{Q}\right] / \Theta$. The space $\widehat{X}$ captures the properties of $\mathbb{k}\left[S_{Q}\right] / \Theta$ more precisely than $X$. On the other hand, $\widehat{X}$ is a manifold with boundary and thus has a geometrical intersection theory. This makes it an object worth studying.

Remark 8.5. The classes $R_{\beta, A}^{\prime} \in\left(E_{X}\right)_{q, q}^{2}$ are the images of the classes $\beta \times\left[T^{(A)}\right] \in$ $H_{q}(\partial Q) \times H_{q}\left(T^{n}\right)$ under the homomorphism $\dot{f}_{*}^{1}:\left(\dot{E}_{\widehat{Y}}\right)_{q, q}^{1} \rightarrow\left(\dot{E}_{\widehat{X}}\right)_{q, q}^{1}$. This homomorphism is injective by Proposition 2.7; thus the construction gives an inclusion

$$
H_{q}(\partial Q) \otimes H_{q}\left(T^{n}\right) \hookrightarrow \operatorname{Soc}\left(\mathbb{k}\left[S_{Q}\right] / \Theta\right)_{2(n-q)}
$$

for each $q \leqslant n-2$. When $q=n-1$, the map $H_{n-1}(\partial Q) \otimes H_{n-1}\left(T^{n}\right) \rightarrow \operatorname{Soc}\left(\mathbb{k}\left[S_{Q}\right] / \Theta\right)_{2}$ has kernel of the form $\langle[\partial Q]\rangle \otimes H_{n-1}\left(T^{n}\right)$, where $[\partial Q]$ is the fundamental class of $\partial Q$. Note that $\partial Q$ may be disconnected, and thus there could exist classes in $H_{n-1}(\partial Q)$ different from $[\partial Q]$. Nevertheless, there exists an injective map

$$
\left(H_{n-1}(\partial Q) /\langle[\partial Q]\rangle\right) \otimes H_{n-1}\left(T^{n}\right) \hookrightarrow \operatorname{Soc}\left(\mathbb{k}\left[S_{Q}\right] / \Theta\right)_{2} .
$$

These statements reprove the result of Novik and Swartz [11, Th. 3.5] in the case of homology manifolds.

\section{References}

[1] A. Ayzenberg, Locally standard torus actions and sheaves over Buchsbaum posets, preprint arXiv:1501.04768.

[2] A. Ayzenberg, Locally standard torus actions and $h^{\prime}$-vectors of simplicial posets, preprint arXiv:1501.07016. To appear in J. Math. Soc. Japan. 
[3] A. Ayzenberg, M. Masuda, S. Park, and H. Zeng, Cohomology of toric origami manifolds with acyclic proper faces, preprint arXiv:1407.0764. To appear in J. Symplectic Geom.

[4] R.A. Brualdi, and H. Schneider, Determinantal identities: Gauss, Schur, Cauchy, Sylvester, Kronecker, Jacobi, Binet, Laplace, Muir, and Cayley, Linear Algebra Appl. 52/53 (1983), 769-791.

[5] V.M. Buchstaber, and T.E. Panov, Combinatorics of simplicial cell complexes and torus actions, Proc. Steklov Inst. Math. 247 (2004), 1-17.

[6] V. Buchstaber, and T. Panov, Toric Topology, Mathematical Surveys and Monographs, 204, American Mathematical Society, Providence, RI, 2015.

[7] A. Cannas da Silva, V. Guillemin, and A.R. Pires, Symplectic origami, Int. Math. Res. Not. IMRN (2011), 4252-4293.

[8] M. Davis, and T. Januszkiewicz, Convex polytopes, Coxeter orbifolds and torus actions, Duke Math. J. 62 (1991), no. 2, 417-451.

[9] M. Masuda, and T. Panov, On the cohomology of torus manifolds, Osaka J. Math. 43 (2006), 711-746.

[10] M. Masuda and S. Park, Toric origami manifolds and multi-fans, Proc. Steklov Inst. Math. 286 (2014), 308-323.

[11] I. Novik, and E. Swartz, Socles of Buchsbaum modules, complexes and posets, Adv. Math. 222 (2009), 2059-2084.

[12] M. Poddar, and S. Sarkar, A class of torus manifolds with nonconvex orbit space, Proc. Amer. Math. Soc. 143 (2015), 1797-1811.

[13] C.P. Rourke, and B.J. Sanderson, Introduction to piecewise-linear topology, Springer Study Edition 69, 1982.

[14] P. Schenzel, On the number of faces of simplicial complexes and the purity of Frobenius, Math. Z. 178 (1981), 125-142.

[15] R.P. Stanley, f-vectors and h-vectors of simplicial posets, J. Pure Appl. Algebra 71(1991), 319-331.

[16] T. Yoshida, Local torus actions modeled on the standard representation, Adv. Math. 227 (2011), 1914-1955.

Anton Ayzenberg ayzenberga@gmail.com

Faculty of Mathematics, Higher School of Economics, 7 Vavilova Str., Moscow, 117312, Russia 